\title{
Position-velocity diagrams of ionized gas in the inner regions of disk galaxies ${ }^{\star}$
}

\author{
J. G. Funes, S. J. ${ }^{1}$, E. M. Corsini ${ }^{2}$, M. Cappellari ${ }^{3, \star \star}$, A. Pizzella ${ }^{4}$, J. C. Vega Beltrán $^{5}$, \\ C. Scarlata ${ }^{4,6}$, and F. Bertola ${ }^{4}$ \\ 1 Vatican Observatory, University of Arizona, Tucson, AZ 85721, USA \\ 2 Osservatorio Astrofisico di Asiago, Dipartimento di Astronomia, Università di Padova, via dell'Osservatorio 8, \\ 36012 Asiago, Italy \\ 3 Leiden Observatory, Postbus 9513, 2300 RA Leiden, The Netherlands \\ 4 Dipartimento di Astronomia, Università di Padova, vicolo dell'Osservatorio 2, 35122 Padova, Italy \\ 5 Instituto Astrofísico de Canarias, Calle Via Lactea s/n, 38200 La Laguna, Spain \\ 6 Space Telescope Science Institute, 3700 San Martin Dr., Baltimore, MD 21218, USA
}

Received 12 December 2001 / Accepted 18 March 2002

\begin{abstract}
We use long-slit spectroscopy along the major axis of a sample of 23 nearby disk galaxies to study the kinematic properties of the ionized-gas component in their inner regions. For each galaxy, we derive the position-velocity diagram of the ionized gas from its emission lines. We discuss the variety of shapes observed in such position-velocity diagrams by comparing the gas velocity gradient, velocity dispersion and integrated flux measured in the inner $\left(r \simeq \pm 1^{\prime \prime}\right)$ and outer regions $\left(r \simeq \pm 4^{\prime \prime}\right)$. This kind of analysis allows the identification of galaxies which are good candidates to host a circumnuclear Keplerian gaseous disk rotating around a central mass concentration, and to follow up with Hubble Space Telescope observations.
\end{abstract}

Key words. galaxies: kinematics and dynamics - galaxies: ISM - galaxies: spiral - galaxies: structure galaxies: nuclei - black hole physics

\section{Introduction}

It is now commonly accepted that supermassive black holes (SMBHs hereafter) are nearly ubiquitous in elliptical galaxies and bulges. According to the standard paradigm, they should constitute the relics of the intense quasar activity that occurred in the early phase of galaxy evolution (see Ho 1999 for a review). The study of SMBHs has greatly benefited from the high resolution capabilities of the spectrographs onboard the Hubble Space Telescope (HST). Nowadays about 40 galaxies, belonging to different morphological types are known to harbor a SMBH.

\footnotetext{
Send offprint requests to: J. G. Funes, S. J.,

e-mail: jfunes@as.arizona.edu

* Based on observations carried out at European Southern Observatory (ESO N.58, A-0564), at the Multiple Mirror Telescope, which is a joint facility of the Smithsonian Institution and the University of Arizona, and at the Isaac Newton Telescope operated by the Isaac Newton group at the La Palma island at the Spanish Observatorio del Roque de los Muchachos of the Instituto de Astrofisica de Canarias.

$\star \star$ ESA external fellow.
}

Their masses, $M_{\bullet}$, range from $10^{6}$ to $10^{10} M_{\odot}$ and have been derived from the analysis of the stellar orbital structure or from the dynamics of gaseous circumnuclear Keplerian disks (CNKD), using both optical and radio observations (see Kormendy \& Gebhardt 2001 and Merritt \& Ferrarese 2001b for both the object list and a discussion of the accuracy of $M_{\bullet}$ determinations).

The census of SMBHs is now large enough to probe the links between $M_{\bullet}$ and the global properties of the host galaxies. $M_{\bullet}$ correlates with the luminosity, $L_{\mathrm{sph}}$, and velocity dispersion, $\sigma$, of the spheroidal component of the host galaxy (Kormendy \& Richstone 1995; Magorrian et al. 1998; Ferrarese \& Merritt 2000; Gebhardt et al. 2000a). A lively debate is ongoing about the slope of the $M_{\bullet}-\sigma$ relation (Merritt \& Ferrarese 2001a), although with its neglegible scatter it is a tighter correlation than the $M_{\bullet}-L_{\mathrm{sph}}$ relation. The consistency of $M_{\bullet}$ in active and quiescent galaxies has been discussed by different authors. Gebhardt et al. (2000b) and Ferrarese et al. (2001) showed that SMBH masses from reverberation mapping agree with the $M_{\bullet}-\sigma$ relation. Similarly, McLure \& Dunlop (2001, 2002) found that the values of $M_{\bullet}$ inferred from 
$L_{\mathrm{sph}}$ for a large sample of quasars and Seyfert galaxies agree with those inferred from $\sigma$ measurements. Elliptical and disk galaxies seem to follow the same $M_{\bullet}-L_{\mathrm{sph}}$ and $M_{\bullet}-\sigma$ correlations, suggesting a close connection between the processes leading to the growth of central SMBHs and the formation of galaxy spheroids, whether they are ellipticals, classical bulges or pseudobulges (Kormendy 2001). Morever, $M_{\bullet}$ does not correlate with disks as it does with spheroids. To date, however, dynamical SMBH detections are available for only a dozen disk galaxies, and, therefore, the addition of new $M_{\bullet}$ determinations for S0's and spirals is highly desirable.

Over the course of the last few years, we have undertaken a vast program aimed at detecting CNKDs in disk galaxies using ground-based spectroscopic observations. Our goal is to measure upper limits for SMBH masses by using HST spectra to constrain them further (Bertola et al. 1998). Here we present a survey of the ionized-gas kinematics of the inner regions of 23 disk galaxies. The study complements the recent results regarding rapidly rotating gaseous nuclear disks in Rubin et al. (1997) and Sofue et al. (1998). The paper is organized as follows: in Sect. 2 we give an overview of the global properties of the sample galaxies and discuss observations and data reduction. In Sect. 3 we derive the position-velocity $(P V)$ diagrams of the emission lines for each sample galaxy, suggesting a scheme for their classification. In Sect. 4 we present our conclusions. Relevant properties and the $P V$ diagram of individual galaxies are discussed in the appendix.

\section{Sample selection, spectroscopic observations and data reduction}

All the observed galaxies are bright $\left(B_{\mathrm{T}} \leq 13.5\right)$ and nearby $\left(V_{\odot}<5800 \mathrm{~km} \mathrm{~s}^{-1}\right)$ with an intermediate-to-high inclination $\left(45^{\circ} \leq i \leq 80^{\circ}\right)$. The Hubble morphological types of the sample galaxies range from S0 to Sd and 5 objects are barred or weakly barred (RC3). The galaxies have been chosen to have strong emission lines. An overview of their basic properties is given in Table 1 . In Fig. 1 we show the absolute magnitude distribution for the galaxies in our sample. The distribution brackets the $M^{*}$ value for spiral galaxies taken from Marzke et al. (1998) for $H_{0}=75 \mathrm{~km} \mathrm{~s}^{-1} \mathrm{Mpc}^{-1}$.

The long-slit spectroscopic observations of our sample galaxies were carried out at the 4.5-m Multiple Mirror Telescope (MMT) in Arizona (USA), at the 3.6-m ESO Telescope at La Silla (Chile), and at the 2.5-m Isaac Newton Telescope (INT) at La Palma (Spain). Details about the instrumental setup and the seeing of each observing run are summarized in Table 2 .

At the beginning of each exposure, the slit was centered on the galaxy nucleus and aligned along the galaxy major axis using the guide TV camera. The slit orientation and the exposure times are given in Table 3. A comparison-lamp spectrum was obtained before and after each object integration to allow an accurate

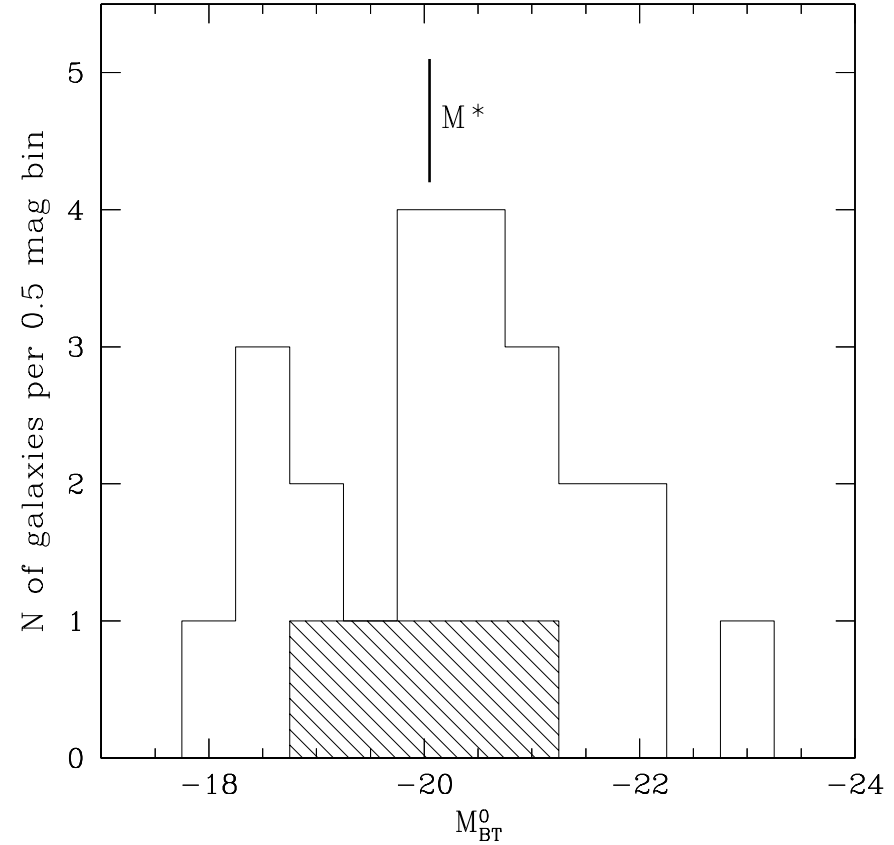

Fig. 1. Absolute magnitude distribution for the sample galaxies. A line marks $M_{B_{\mathrm{T}}}^{0}=-20.05$, which corresponds to $M^{*}$ for spiral galaxies as derived by Marzke et al. (1998) and assuming $H_{0}=75 \mathrm{~km} \mathrm{~s}^{-1} \mathrm{Mpc}^{-1}$. The dashed region identifies galaxies classified barred or weakly barred in RC3.

wavelength calibration. Quartz-lamp and twilight-sky flatfields were taken to map pixel-to-pixel sensitivity variations and large-scale illumination patterns.

Using standard MIDAS $^{1}$ routines, the spectra were bias subtracted, flatfield corrected, cleaned for cosmic rays, and wavelength calibrated. Cosmic rays were identified by comparing the counts in each pixel with the local mean and standard deviation (based on the Poisson statistics of the photons are using the gain and readout noise of the detector). We corrected the data by interpolating a suitable value.

The instrumental resolution was derived as the mean of the Gaussian FWHMs measured for a number of unblended arc-lamp lines (12 in the MMT and INT spectra and 30 in the ESO spectra), which were distributed over the whole spectral range of a wavelength-calibrated comparison spectrum. The mean FWHM of the arc-lamp lines, as well as the corresponding instrumental velocity dispersion, are given in Table 2. Finally, the spectra were aligned and coadded using the centers of their stellar continua as reference. In the resulting spectra, the contribution from the sky was determined by interpolating along the outermost $10^{\prime \prime}-20^{\prime \prime}$ at the edges of the slit, where galaxy light was negligible. The sky level was then subtracted.

1 MIDAS is developed and maintained by the European Southern Observatory. 
Table 1. Parameters of the sample galaxies.

\begin{tabular}{|c|c|c|c|c|c|c|c|c|c|c|c|c|c|}
\hline $\begin{array}{c}\text { Object } \\
\text { [name] } \\
(1)\end{array}$ & $\begin{array}{c}\text { Morp. } \\
{[\mathrm{RSA}]} \\
(2)\end{array}$ & $\begin{array}{l}\text { ype } \\
\text { [RC3] } \\
(3)\end{array}$ & $\begin{array}{c}B_{\mathrm{T}} \\
{[\mathrm{mag}]} \\
(4)\end{array}$ & $\begin{array}{l}\mathrm{PA} \\
{\left[^{\circ}\right]} \\
(5)\end{array}$ & $\begin{array}{c}i \\
{\left[^{\circ}\right]} \\
(6)\end{array}$ & $\begin{array}{c}V_{\odot} \\
{\left[\mathrm{km} \mathrm{s}^{-1}\right]} \\
(7)\end{array}$ & $\begin{array}{c}D \\
{[\mathrm{Mpc}]} \\
(8)\end{array}$ & $\begin{array}{c}\text { Scale } \\
{\left[\mathrm{pc} /{ }^{\prime \prime}\right]} \\
(9)\end{array}$ & $\begin{array}{c}M_{B_{\mathrm{T}}}^{0} \\
{[\mathrm{mag}]} \\
(10)\end{array}$ & $\begin{array}{c}\sigma_{\star} \\
{\left[\mathrm{km} \mathrm{s}^{-1}\right]} \\
(11)\end{array}$ & $\begin{array}{c}M_{\bullet} \\
{\left[M_{\odot}\right]} \\
(12)\end{array}$ & $\begin{array}{c}\theta_{\bullet} \\
{\left[{ }^{\prime \prime}\right]} \\
(13)\end{array}$ & $\begin{array}{c}\text { Nuc. Type } \\
(14)\end{array}$ \\
\hline NGC 470 & Sbc(s)II.3 & .SAT3.. & 12.53 & 155 & 52 & 2370 & 33.8 & 163.9 & -20.66 & 56 & $3.2 \mathrm{e}+05$ & 0.003 & $\ldots$ \\
\hline NGC 772 & $\mathrm{Sb}(\mathrm{rs}) \mathrm{I}$ & .SAS3.. & 11.09 & 130 & 54 & 2470 & 35.6 & 172.7 & -22.21 & 124 & $1.4 \mathrm{e}+07$ & 0.02 & $\ldots$ \\
\hline NGC 949 & $\mathrm{Sc}(\mathrm{s}) \mathrm{III}$ &. $\mathrm{SAT}^{*} * \$$ & 12.40 & 145 & 58 & 620 & 11.4 & 55.2 & -18.50 & 32 & $2.3 \mathrm{e}+04$ & 0.002 & $\ldots$ \\
\hline NGC 980 & $\ldots$ & $. \mathrm{L} \ldots . .$. & 13.16 & 110 & 58 & 5765 & 80.1 & 388.2 & -22.95 & 226 & $2.3 \mathrm{e}+08$ & 0.06 & $\ldots$ \\
\hline NGC 1160 & $\ldots$ & S...6*. & 13.50 & 50 & 62 & 2510 & 36.6 & 177.4 & -21.01 & 24 & $5.9 \mathrm{e}+04$ & 0.0003 & $\ldots$ \\
\hline NGC 2179 & $\mathrm{Sa}$ & .SAS0.. & 13.22 & 170 & 47 & 2885 & 36.5 & 177.0 & -19.98 & 166 & $5.4 \mathrm{e}+07$ & 0.05 & $\ldots$ \\
\hline NGC 2541 & $\mathrm{Sc}(\mathrm{s}) \mathrm{III}$ & .SAS6.. & 12.26 & 165 & 61 & 565 & 8.7 & 42.2 & -18.13 & 53 & $2.5 \mathrm{e}+05$ & 0.01 & $\mathrm{~T} 2 / \mathrm{H}:$ \\
\hline NGC 2683 & $\mathrm{Sb}$ (on edge) & .SAT3.. & 10.64 & 44 & 78 & 400 & 5.3 & 25.6 & -18.99 & 83 & $2.0 \mathrm{e}+06$ & 0.06 & $\mathrm{~L} 2 / \mathrm{S} 2$ \\
\hline NGC 2768 & $\mathrm{~S}_{1 / 2}(6)$ & .E.6.* & 10.84 & 95 & 59 & 1331 & 19.4 & 94.1 & -20.74 & 205 & $1.5 \mathrm{e}+08$ & 0.2 & L2 \\
\hline NGC 2815 & $\mathrm{Sb}(\mathrm{s}) \mathrm{I}-\mathrm{II}$ & .SBR3* & 12.81 & 10 & 72 & 2541 & 30.5 & 147.7 & -21.00 & 168 & $5.7 \mathrm{e}+07$ & 0.07 & $\mathrm{~L} / \mathrm{S} 2$ \\
\hline NGC 2841 & $\mathrm{Sb}$ & SAR3* & 10.09 & 147 & 65 & 640 & 9.6 & 46.4 & -20.33 & 197 & $1.2 \mathrm{e}+08$ & 0.3 & L2 \\
\hline NGC 3031 & $\mathrm{Sb}(\mathrm{r}) \mathrm{I}-\mathrm{II}$ & .SAS2.. & 7.89 & 157 & 59 & -50 & 1.5 & 7.2 & -18.46 & 173 & $6.6 \mathrm{e}+07$ & 1.5 & S1.5 \\
\hline NGC 3281 & $\mathrm{Sa}$ &. $\mathrm{SAS} 2 \mathrm{P}^{*}$ & 12.70 & 140 & 61 & 3380 & 41.1 & 199.5 & -21.25 & 172 & $6.4 \mathrm{e}+07$ & 0.05 & $\mathrm{~S} 2$ \\
\hline NGC 3368 & $\operatorname{Sab}(\mathrm{s}) \mathrm{II}$ & .SXT2.. & 10.11 & 5 & 47 & 865 & 9.7 & 47.1 & -20.14 & 129 & $1.6 \mathrm{e}+07$ & 0.1 & $\mathrm{~L} 2$ \\
\hline NGC 3521 & $\mathrm{Sb}(\mathrm{s}) \mathrm{II}-\mathrm{III}$ & .SXT4.. & 9.04 & 163 & 63 & 825 & 8.5 & 41.1 & -20.35 & 145 & $2.8 \mathrm{e}+07$ & 0.2 & H/L2: \\
\hline NGC 3705 & $\operatorname{Sab}(r) I-I I$ & .SXR2.. & 11.86 & 122 & 66 & 1000 & 11.4 & 55.2 & -19.03 & 109 & $7.4 \mathrm{e}+06$ & 0.05 & $\mathrm{~T} 2$ \\
\hline NGC 3898 & $\mathrm{SaI}$ & .SAS2.. & 11.60 & 107 & 54 & 1184 & 17.1 & 82.9 & -19.85 & 223 & $2.2 \mathrm{e}+08$ & 0.3 & $\mathrm{~T} 2$ \\
\hline NGC 4419 & SBab: & .SBS1./ & 12.08 & 133 & 71 & -210 & 17.0 & 82.4 & -19.55 & 98 & $4.5 \mathrm{e}+06$ & 0.03 & $\mathrm{H}$ \\
\hline NGC 4698 & $\mathrm{Sa}$ & .SAS2.. & 11.46 & 170 & 52 & 992 & 17.0 & 82.4 & -19.91 & 174 & $6.7 \mathrm{e}+07$ & 0.1 & $\mathrm{~S} 2$ \\
\hline NGC 5064 & $\mathrm{Sa}$ & PSA. $2^{*}$ & 13.04 & 38 & 63 & 2980 & 36.0 & 174.4 & -21.11 & 202 & $1.4 \mathrm{e}+08$ & 0.09 & $\mathrm{~L}$ \\
\hline NGC 7320 & $\ldots$ & .SAS7.. & 13.23 & 132 & 60 & 862 & 15.4 & 74.7 & -18.39 & $\ldots$ & $\ldots$ & $\ldots$ & $\ldots$ \\
\hline NGC 7331 & $\mathrm{Sb}(\mathrm{rs}) \mathrm{I}-\mathrm{II}$ & .SAS3.. & 10.35 & 171 & 70 & 820 & 14.7 & 72.0 & -21.48 & 141 & $2.5 \mathrm{e}+07$ & 0.09 & $\mathrm{~T} 2$ \\
\hline NGC 7782 & $\mathrm{Sb}(\mathrm{s}) \mathrm{I}-\mathrm{II}$ & .SAS3.. & 13.08 & 175 & 58 & 5430 & 75.3 & 364.9 & -21.95 & 193 & $1.1 \mathrm{e}+08$ & 0.04 & $\ldots$ \\
\hline
\end{tabular}

Notes - Column 2: morphological classification from RSA. Column 3: morphological classification from RC3. Column 4: total observed blue magnitude from RC3 except for NGC 980 and NGC 5064 (LEDA). Column 4: major-axis position angle taken from RC3. Column 6 : inclination derived from cos ${ }^{2} i=\left(q^{2}-q_{0}^{2}\right) /\left(1-q_{0}^{2}\right)$. The observed axial ratio $q$ is taken from RC3 and the intrinsic flattening $q_{0}=0.11$ has been assumed following Guthrie (1992). Column 7: heliocentric velocity of the galaxy derived from the center of symmetry of the rotation curve of the gas. They are taken from Vega Beltrán et al. (2001) except for NGC 2179, NGC 3281 and NGC 4698 (Corsini et al. 1999). Column 8: distance obtained as $V_{0} / H_{0}$ with $H_{0}=75 \mathrm{~km} \mathrm{~s}^{-1} \mathrm{Mpc}^{-1}$ and $V_{0}$ the systemic velocity derived from $V_{\odot}$ corrected for the motion of the Sun with respect of the Local Group according to the RSA. For NGC 4419 and NGC 4698, which belong to the Virgo cluster, we assume a distance of 17 Mpc (Freedman et al. 1994 ). Column 10: absolute total blue magnitude corrected for inclination and extinction from RC3. Column 11: central velocity dispersion of the stellar component. Data are from Vega Beltrán et al. (2001) except for NGC 2179, NGC 3281 (Corsini et al. 1999), NGC 2768 (Bertola et al. 1995), NGC 2815 (LEDA), NGC 3521 (Zeilinger et al. 2001), and NGC 4698 (Bertola et al. 1999). Column 12: expected mass of the central SMBH derived from $\sigma_{\star}$ following Merritt \& Ferrarese (2001a). Column 13: angular size of the radius of influence of the central SMBH derived from $\theta_{\bullet} \approx M_{\bullet} \sigma_{\star}^{-2} D^{-1}$ (de Zeeuw 2001), where $M_{\bullet}$ is the mass of the SMBH in units of $10^{6} M_{\odot}, \sigma_{\star}$ is the central velocity dispersion in units of $100 \mathrm{~km} \mathrm{~s}^{-1}$, and $D$ is the galaxy distance in Mpc. Column 14: classification of the nuclear spectrum from Ho et al. (1997) except NGC 2815 (Véron-Cetty \& Véron 1986). H = H II nucleus. L = LINER. S = Seyfert nucleus. $\mathrm{T}=$ transition object. ... = uncertain. 
Table 2. Instrumental setup for spectroscopic observations.

\begin{tabular}{|c|c|c|c|}
\hline Parameter & $\overline{\mathrm{MMT}}$ & $\overline{\text { INT }}$ & 3.6-m ESO \\
\hline Date & $21-23$ Oct. $1990 \quad 17-18$ Dec. 1990 & 19-20 Mar. 1996 & 03-04 Feb. 1997 \\
\hline Spectrograph & Red Channel $^{\mathrm{a}}$ & $\operatorname{IDS}^{\mathrm{a}}$ & CASPEC $^{\mathrm{b}}$ \\
\hline Grating (grooves $\mathrm{mm}^{-1}$ ) & 1200 & 1800 & 31.6 \\
\hline Detector & Loral $12 \times 8 \mathrm{mmt}$ & TK1024A & TK1024AB \\
\hline Pixel size $\left(\mu \mathrm{m}^{2}\right)$ & $15 \times 15$ & $24 \times 24$ & $24 \times 24$ \\
\hline Pixel binning & $1 \times 1$ & $1 \times 1$ & $1 \times 1$ \\
\hline Scale (" pixel $\left.{ }^{-1}\right)$ & 0.30 & 0.33 & 0.33 \\
\hline Reciprocal dispersion $\left(\AA\right.$ pixel $\left.^{-1}\right)$ & 0.82 & 0.24 & 0.076 \\
\hline Slit width $\left({ }^{\prime \prime}\right)$ & 1.25 & 1.9 & 1.3 \\
\hline Slit length $\left({ }^{\prime}\right)$ & 3.0 & 4.0 & 2.4 \\
\hline Spectral range $(\AA)$ & $4850-5500$ & $6650-6890$ & $6630-6651$ \\
\hline Comparison lamp & $\mathrm{He}-\mathrm{Ne}-\mathrm{Ar}-\mathrm{Fe}$ & $\mathrm{Cu}-\mathrm{Ar}$ & Th-Ar \\
\hline Instrumental $F W H M(\AA)$ & $2.57 \pm 0.11$ & $0.869 \pm 0.040$ & $0.233 \pm 0.017$ \\
\hline Instrumental $\sigma^{\mathrm{c}}\left(\mathrm{km} \mathrm{s}^{-1}\right)$ & 57 & 5 & 17 \\
\hline Seeing $F W H M\left({ }^{\prime \prime}\right)$ & $1.2-1.5$ & $1.0-1.4$ & $0.8-1.2$ \\
\hline
\end{tabular}

a Grating used at the first order.

b CASPEC mounting of the Long Camera in long-slit configuration without crossdisperser. The spectral order \#86 ( $\lambda_{\mathrm{c}}=6617 \AA$ ) corresponding to the redshifted $\mathrm{H} \alpha$ region was isolated by means of the narrow-band $6630 / 51 \AA$ filter.

c The instrumental dispersion has been measured at [O III] $\lambda 5007$, [N II] $\lambda 6583$ and $\mathrm{H} \alpha$ for the MMT, INT and 3.6-m ESO spectra, respectively.

Table 3. Log of spectroscopic observations.

\begin{tabular}{|c|c|c|c|c|}
\hline $\begin{array}{l}\text { Object } \\
\text { [name] }\end{array}$ & $\overline{\text { Date }}$ & Telescope & $\begin{array}{c}t_{\exp } \\
{[\mathrm{s}]}\end{array}$ & $\begin{array}{c}\mathrm{PA} \\
\left.{ }^{\circ}\right]\end{array}$ \\
\hline NGC 470 & 22 Oct. 90 & MMT & 3600 & 155 \\
\hline NGC 772 & 22 Oct. 90 & MMT & 3600 & 130 \\
\hline NGC 949 & 21 Oct. 90 & MMT & 3600 & 145 \\
\hline NGC 980 & 22 Oct. 90 & MMT & 3600 & 110 \\
\hline NGC 1160 & 21 Oct. 90 & MMT & 3600 & 50 \\
\hline \multirow[t]{2}{*}{ NGC 2179} & 03 Feb. 97 & 3.6-m ESO & $4 \times 3600$ & 170 \\
\hline & 04 Feb. 97 & 3.6-m ESO & $2 \times 3600$ & 170 \\
\hline NGC 2541 & 21 Oct. 90 & MMT & 3600 & 165 \\
\hline NGC 2683 & 18 Dec. 90 & MMT & 3600 & 44 \\
\hline NGC 2768 & 19 Mar. 96 & INT & $2 \times 3600$ & 95 \\
\hline NGC 2815 & 04 Feb. 97 & 3.6-m ESO & $3 \times 3600$ & 10 \\
\hline NGC 2841 & 22 Oct. 90 & MMT & 3600 & 147 \\
\hline NGC 3031 & 17 Dec. 90 & MMT & 3600 & 157 \\
\hline NGC 3281 & 04 Feb. 97 & 3.6-m ESO & 3600 & 140 \\
\hline NGC 3368 & 17 Dec. 90 & MMT & 3600 & 5 \\
\hline NGC 3521 & 17 Dec. 90 & MMT & 3600 & 342 \\
\hline NGC 3705 & 17 Dec. 90 & MMT & 3600 & 122 \\
\hline NGC 3898 & 19 Mar. 96 & INT & $3 \times 3600$ & 107 \\
\hline \multirow[t]{2}{*}{ NGC 4419} & 20 Mar. 96 & INT & $2 \times 3300$ & 133 \\
\hline & 20 Mar. 96 & INT & 3600 & 133 \\
\hline NGC 4698 & 20 Mar. 96 & INT & 3600 & 170 \\
\hline \multirow[t]{2}{*}{ NGC 5064} & 03 Feb. 97 & 3.6-m ESO & $3 \times 3600$ & 38 \\
\hline & 04 Feb. 97 & 3.6-m ESO & 3600 & 38 \\
\hline NGC 7320 & 22 Oct. 90 & MMT & 3600 & 132 \\
\hline NGC 7331 & 22 Oct. 90 & MMT & 3600 & 171 \\
\hline NGC 7782 & 22 Oct. 90 & MMT & 3600 & 30 \\
\hline
\end{tabular}

\section{Position-velocity diagrams}

\subsection{Galaxy continuum subtraction}

We subtracted the stellar continua from the spectra to study the two-dimensional shape of the emission lines. Without a large library of stellar (or even galaxy) absorption-line spectra obtained with the same observing setup of the spectra of the sample galaxies we were unable to apply the technique of template subtraction for an optimal correction for the starlight contamination (e.g. Ho et al. 1997).

The galaxy continua have been removed from MMT spectra by applying the technique outlined by Bender et al. (1994). The following procedure was applied to the each row of the galaxy spectrum. First we fit a sixthto-tenth-order polynomial to the observed spectrum and calculated the rms variation, $\sigma$, of the spectrum around the polynomial. Then, the fit was repeated including only those pixels with values falling within $-1 \sigma$ to $0 \sigma$ of the first fit in order to avoid both emission and strong absorption lines. The new polynomial fit was adopted as the galaxy continuum and subtracted from the observed spectrum.

We were prevented from adopting the same method for the INT and ESO spectra because of their shorter wavelength range. Our major concern, with the stellar continuum subtraction was avoiding the creation of spurious features. For this reason we adopted a very simple but robust approach. Specifically, we made the reasonable assumption that the underlying observed stellar profile is the same at all wavelengths in the small observed range. An average profile was determined in regions free from emission-line flux and this same profile, properly scaled and subtracted from all the columns of the spectrum. The stellar continuum under the emission features was approximated by linear interpolation.

For our purposes, the above techniques give a satisfactory approximation of the galaxy continuum in the spectral range centered on the relevant emission lines we 
measure, specifically [O III] $\lambda 5007$, [N II] $\lambda 6583$, and $\mathrm{H} \alpha$ for the MMT, INT and ESO spectra, respectively. In Fig. 2 we show the continuum-subtracted spectra of the sample galaxies as well as the isodensity contour plots (i.e. the $P V$ diagram) of the emission lines we measure.

\subsection{Measuring the position-velocity diagrams}

The $P V$ diagrams plotted in Fig. 2 show the variety of two-dimensional shapes of the optical emission lines in the inner regions of disk galaxies. This finding is in agreement with the earlier results of Rubin et al. (1997) and Sofue et al. (1998). We suggest a classification of these $P V$ diagrams based on the comparison of the velocity gradients, velocity dispersions and fluxes measured at different distances from the center. Our goal is to identify galaxies hosting a CNKD.

We measured the line-of-sight velocity, $V$, of the ionized gas at $r \simeq \pm 1^{\prime \prime}$ and $r \simeq \pm 4^{\prime \prime}$ by fitting a Gaussian to the relevant emission line. The central wavelength of the Gaussian fit was converted to velocity in the optical convention and then the standard heliocentric correction was applied to obtain $V$. The radii, $r \simeq \pm 1^{\prime \prime}$, used for measuring the inner velocity gradient $(\Delta V / \Delta r)_{\text {in }}$, are dictated by the spatial resolution limit imposed by seeing on our data $\left(0.0^{\prime \prime} 8 \lesssim F W H M \lesssim 1^{\prime \prime} .5\right)$. Choosing the smallest possible radii according to the seeing limit assures us that if a central mass concentration is present, the observed inner velocity gradient is maximized. The outer velocity gradient, $(\Delta V / \Delta r)_{\text {out }}$, measured at $r \simeq \pm 4^{\prime \prime}$, serves as a reference. In fact, for each sample galaxy the radius of influence, $\theta_{\bullet}$, of the possible central mass concentration predicted using the $M_{\bullet}-\sigma$ relation (Merritt \& Ferrarese 2001a, see Table 1$)$ is $\theta_{\bullet} \ll 4^{\prime \prime}$. Therefore, $(\Delta V / \Delta r)_{\text {out }}$ is essentially determined by the contribution of galaxy stellar component to the potential.

We checked that velocity gradients did not significatively change if the $\Delta V$ are computed from the difference of line-of-sight velocity distribution maxima instead of the centers of the fitting Gaussian fit. Moreover, to test the robustness of our measurements and to estimate the associated uncertainties, we compute $(\Delta V / \Delta r)_{\text {in }}$ at $r_{\text {in }} \simeq \pm 0.7$ and at $\pm 1^{\prime \prime} .3$ and we compute $(\Delta V / \Delta r)_{\text {out }}$ at $r_{\text {out }} \simeq \pm 3^{\prime \prime}$ and at $\pm 5^{\prime \prime}$, respectively. The errors on $(\Delta V / \Delta r)_{\text {in }}$ have been estimated from the maximum difference between the values measured at \pm 0 ". 7 and \pm 1 ". 3 with respect to those measured at $\pm 1^{\prime \prime}$. Similarly, the errors on $(\Delta V / \Delta r)_{\text {out }}$ have been estimated from the maximum difference between the values obtained at $\pm 3^{\prime \prime}$ and $\pm 5^{\prime \prime}$ with respect to those measured at $\pm 4^{\prime \prime}$. The measured values of $(\Delta V / \Delta r)_{\text {in }}$ and $(\Delta V / \Delta r)_{\text {out }}$ are given in Table 4 .

The inner-to-outer velocity gradient ratio $\Gamma$ is independent of galaxy inclination and has been adopted to indicate which galaxies are characterized by rapidly-rotating gas in the inner regions. However, to allow a direct comparison of their absolute values, we plotted the inner velocity gradients as a function of the corresponding outer velocity gradients in Fig. 3, after correcting for galaxy inclination and distance given in Table 1. NGC 980, NGC 2179, NGC 2683, NGC 3031 and NGC 7782 are clearcut cases of galaxies characterized by $\Gamma \pm \Delta \Gamma>2$.

To characterize the velocity-dispersion and surfacebrightness radial profiles of each gaseous disk, we measured the velocity dispersion and integrated flux of the ionized gas in the galaxy center and at $r \simeq \pm 4^{\prime \prime}$, using a Gaussian fit to the line adopted for the velocity measurements. The FWHM of Gaussian fit was corrected for instrumental FWHM and converted into the velocity dispersion, $\sigma$. The formal error of the fit has been adopted as the error on the central value of velocity dispersion, while the errors on the outer values have been estimated using the maximum difference between the measurements obtained at $\pm 3^{\prime \prime}$ and $\pm 5^{\prime \prime}$ with respect to those at $\pm 4^{\prime \prime}$. The integrated flux was assumed to be the area of the Gaussian fit and the associated error was estimated from Poisson statistics. We considered only the central-to-outer integrated-flux ratio since spectra were not flux calibrated. This process results in line fluxes of different objects observed with different setups that are not directly comparable. The measured values of the velocity dispersion and the central-to-outer integrated-flux ratio are given in Table 4 . The central velocity dispersions are shown as a function of the outer velocity dispersions in Fig. 3. NGC 980, NGC 2179, and NGC 3031 exhibit the sharpest rises in observed velocity dispersion towards their centers.

\subsection{A classification of the position-velocity diagrams}

We propose an operational classification of the $P V$ diagrams of Fig. 2 based on the properties we measured in Sect. 3.2 and based on the analogy between the shapes of the $P V$ diagrams we observed and the shapes predicted for the spectrum of a ionized thin gaseous disk, under the assumption that the gas moves in circular orbits, in the plane of the galaxy. Although the model assumptions may not be accurate in practice, the classification is useful to identify the effects of rapid gas rotation. We do not pretend to draw any general conclusions on the phenomenology of $P V$ diagrams from such a classification; it is just a tool adopted to select the sample galaxies which possibly host CNKDs.

Under the model assumptions, two parameters are crucial in determining the observed shape of the $P V$ diagrams; they are the value of the central mass concentration and the steepness of the intrinsic surface-brightness distribution of the gaseous disk. To investigate the change in the $P V$ diagrams resulting from these two effects, we have used the IDL modeling software developed in Bertola et al. (1998). We refer the reader to that paper for further details on the model. A slit width and a seeing FWHM of $1^{\prime \prime}$ have been adopted, with a spectrograph velocity scale of $10 \mathrm{~km} \mathrm{~s}^{-1}$ pixel $^{-1}$ and a spatial scale of $0^{\prime \prime} 3 \mathrm{pixel}^{-1}$. The underlying galaxy potential is assumed to result in a rigid rotation of $0.4 \mathrm{~km} \mathrm{~s}^{-1} \mathrm{pc}^{-1}$ in the plane of the disk, which is "observed" at $60^{\circ}$ inclination $\left(i=90^{\circ}\right.$ corresponding to 
Table 4. Measured parameters of the sample galaxies.

\begin{tabular}{|c|c|c|c|c|c|c|c|c|}
\hline $\begin{array}{c}\text { Object } \\
\text { [name] } \\
(1) \\
\end{array}$ & $\begin{array}{c}(\Delta V / \Delta r)_{\text {in }} \\
{\left[\mathrm{km} \mathrm{s}^{-1} \operatorname{arcsec}^{-1}\right]} \\
(2)\end{array}$ & $\begin{array}{c}(\Delta V / \Delta r)_{\text {out }} \\
{\left[\mathrm{km} \mathrm{s}^{-1} \operatorname{arcsec}^{-1}\right]} \\
(3)\end{array}$ & $(4)$ & $\begin{array}{c}\sigma_{0} \\
{\left[\mathrm{~km} \mathrm{~s}^{-1}\right]} \\
(5)\end{array}$ & $\begin{array}{c}\sigma_{\text {out }} \\
{\left[\mathrm{km} \mathrm{s}^{-1}\right]} \\
(6)\end{array}$ & $\begin{array}{c}\sigma_{0} / \sigma_{\text {out }} \\
(7)\end{array}$ & $\begin{array}{c}F_{0} / F_{\text {out }} \\
(8) \\
\end{array}$ & $\begin{array}{c}\text { Type } \\
(9) \\
\end{array}$ \\
\hline NGC 470 & $33 \pm 4$ & $23 \pm 7$ & $1.4_{-0.5}^{+0.9}$ & $101 \pm 15$ & $103 \pm 41$ & $1.0_{-0.4}^{+0.9}$ & $4.0 \pm 0.1$ & III \\
\hline NGC 772 & $32 \pm 3$ & $33 \pm 19$ & $1.0_{-0.4}^{+1.5}$ & $131 \pm 19$ & $138 \pm 51$ & $\begin{array}{r}-0.4 \\
0.9_{-0.4}^{+0.8}\end{array}$ & $3.7 \pm 0.1$ & III \\
\hline NGC 949 & $6 \pm 3$ & $6 \pm 4$ & $1.0_{-0.7}^{+3.5}$ & $57 \pm 13$ & $42 \pm 23$ & $1.4_{-0.3}^{+2.4}$ & $2.7 \pm 0.1$ & III \\
\hline NGC 980 & $108 \pm 12$ & $52 \pm 11$ & $2.1_{-0.6}^{+0.8}$ & $259 \pm 34$ & $129 \pm 95$ & $2.0_{-1.0}^{+6.6}$ & $6.9 \pm 0.1$ & I \\
\hline NGC 1160 & $17 \pm 14$ & $33 \pm 24$ & $0.5_{-0.5}^{+2.9}$ & $66 \pm 21$ & $32 \pm 11$ & $2.0_{-1.0}^{+2.0}$ & $1.2 \pm 0.1$ & II \\
\hline NGC 2179 & $86 \pm 4$ & $41 \pm 6$ & $2.1_{-0.4}^{+0.5}$ & $170 \pm 29$ & $42 \pm 11$ & $4.0_{-1.4}^{+2.4}$ & $4.2 \pm 0.1$ & I \\
\hline NGC 2541 & $7 \pm 1$ & $4 \pm 2$ & $1.8_{-0.8}^{+2.3}$ & $48 \pm 18$ & $55 \pm 13$ & $0.9_{-0.4}^{+0.4}$ & $5.2 \pm 0.2$ & III \\
\hline NGC 2683 & $36 \pm 6$ & $12 \pm 8$ & $3.0_{-1.5}^{+7.5}$ & $109 \pm 13$ & $163 \pm 31$ & $0.7_{-0.2}^{+0.3}$ & $3.2 \pm 0.1$ & $*$ \\
\hline NGC 2768 & $20 \pm 2$ & $7 \pm 4$ & $2.9_{-1.2}^{+1.5}$ & $174 \pm 12$ & $141 \pm 48$ & $1.2_{-0.4}^{+0.8}$ & $10.8 \pm 0.1$ & III \\
\hline NGC 2815 & $49 \pm 1$ & $49 \pm 6$ & $1.0_{-0.1}^{+0.2}$ & $149 \pm 16$ & $94 \pm 9$ & $1.6_{-0.3}^{+0.4}$ & $15.0 \pm 0.5$ & III \\
\hline NGC 2841 & $15 \pm 7$ & $17 \pm 2$ & $0.9_{-0.5}^{+0.6}$ & $135 \pm 18$ & $144 \pm 19$ & $0.9_{-0.2}^{+0.3}$ & $2.5 \pm 0.1$ & III \\
\hline NGC 3031 & $21 \pm 4$ & $7 \pm 3$ & $3.0_{-1.3}^{+3.3}$ & $237 \pm 51$ & $57 \pm 41$ & $4.2_{-2.3}^{+13.8}$ & $65.6 \pm 0.4$ & III \\
\hline NGC 3281 & $34 \pm 5$ & $18 \pm 9$ & $1.9_{-0.8}^{+2.4}$ & $119 \pm 20$ & $92 \pm 12$ & $1.3_{-0.3}^{+0.3}$ & $19.9 \pm 0.5$ & III \\
\hline NGC 3368 & $35 \pm 12$ & $32 \pm 8$ & $1.1_{-0.5}^{+0.9}$ & $113 \pm 19$ & $69 \pm 12$ & $1.6_{-0.5}^{+0.3}$ & $6.1 \pm 0.2$ & III \\
\hline NGC 3521 & $21 \pm 10$ & $18 \pm 11$ & $1.2_{-0.8}^{+3.3}$ & $180 \pm 39$ & $146 \pm 66$ & $1.2_{-0.6}^{+0.5}$ & $8.9 \pm 0.1$ & III \\
\hline NGC 3705 & $22 \pm 1$ & $14 \pm 10$ & $1.6_{-0.7}^{+4.2}$ & $110 \pm 14$ & $55 \pm 42$ & $2.0_{-1.0}^{+7.0}$ & $13.3 \pm 0.4$ & III \\
\hline NGC 3898 & $13 \pm 1$ & $26 \pm 3$ & $0.5_{-0.1}^{+0.1}$ & $131 \pm 21$ & $110 \pm 27$ & $1.2_{-0.4}^{+0.6}$ & $4.6 \pm 0.1$ & III \\
\hline NGC 4419 & $7 \pm 1$ & $14 \pm 2$ & $0.5_{-0.1}^{+0.2}$ & $117 \pm 11$ & $83 \pm 21$ & $1.4_{-0.4}^{+0.4}$ & $13.6 \pm 0.1$ & III \\
\hline NGC 4698 & $8 \pm 5$ & $3 \pm 1$ & $2.7_{-1.9}^{+3.8}$ & $86 \pm 8$ & $103 \pm 7$ & $0.8_{-0.1}^{+0.4}$ & $6.0 \pm 0.1$ & III \\
\hline NGC 5064 & $74 \pm 11$ & $56 \pm 11$ & $1.3_{-0.4}^{+0.6}$ & $52 \pm 22$ & $43 \pm 11$ & $1.2_{-0.7}^{+1.1}$ & $0.9 \pm 0.1$ & II \\
\hline NGC 7320 & $6 \pm 1$ & $5 \pm 3$ & $1.2_{-0.6}^{+2.3}$ & $12 \pm 10$ & $10 \pm 7$ & $1.2_{-1.1}^{+6.1}$ & $0.3 \pm 0.1$ & II \\
\hline NGC 7331 & $16 \pm 4$ & $15 \pm 11$ & $1.1_{-0.6}^{+3.9}$ & $102 \pm 11$ & $130 \pm 65$ & $0.8_{-0.3}^{+1.0}$ & $3.7 \pm 0.1$ & III \\
\hline NGC 7782 & $170 \pm 25$ & $56 \pm 11$ & $3.0_{-0.9}^{+1.3}$ & $151 \pm 19$ & $96 \pm 29$ & $1.6_{-0.5}^{+1.0}$ & $5.3 \pm 0.1$ & I \\
\hline
\end{tabular}

Notes - Column 2: inner velocity gradient at $r \simeq \pm 1^{\prime \prime}$. Column 3: outer velocity gradient at $r \simeq \pm 4^{\prime \prime}$. Column 4 : inner-to-outer velocity gradient ratio. Column 5: central velocity dispersion. Column 6: outer velocity dispersion at $r \simeq \pm 4^{\prime \prime}$. Column 7 : centralto-outer velocity dispersion ratio. Column 8: central-to-outer integrated-flux ratio. $F_{0}$ and $F_{\text {out }}$ have been measured at $r=0^{\prime \prime}$ and $r \simeq \pm 4^{\prime \prime}$, respectively. Column 9: type of $P V$ diagram according to our classification; ${ }^{*}=$ figure-of-eight $P V$ diagram (see appendix).

edge-on). A distance of $17 \mathrm{Mpc}$ was adopted for the modeling, corresponding to the distance of the Virgo cluster.

The predicted effect on the $P V$ diagram from an increase of the central mass concentration is presented in the upper panels of Fig. 4. In this case, we assume an exponential the surface-brightness profile superposed on a constant term: $I(R)=I_{0}+I_{1} \exp \left(-R / R_{I}\right)$, with $I_{0}=1$ and $I_{1}=5$ (in arbitrary units) and $R_{I}=1^{\prime \prime}$, where the central mass is given by $M_{\bullet}=0,10^{8}, 10^{9} M_{\odot}$ in panels (a), (b) and $(\mathrm{c})$ respectively.

The $P V$ change that results from a variation in the brightness of a central unresolved source is shown in the bottom panels of Fig. 4. In these panels the adopted surface-brightness profile is assumed to be an essentially unresolved Gaussian superposed on a constant term: $I(R)=I_{0}+I_{1} \exp \left[-R^{2} /\left(2 \sigma_{I}^{2}\right)\right]$, with $I_{0}=1, \sigma_{I}=0^{\prime \prime} 3$ and $I_{1}=0,20,100$ (in arbitrary units) in panel (a), (b) and (c) respectively.

By comparing the models of Fig. 4 with the observed $P V$ diagrams, and inspecting the measured values of $\Gamma$, $\sigma_{0} / \sigma_{\text {out }}$ and $F_{0} / F_{\text {out }}$ we identify three different types of $P V$ diagrams (see Fig. 5).

Type I. This type of $P V$ diagrams suggests the presence of two distinct kinematical gaseous components. This results from the sharp increase of $\Delta V / \Delta r$ towards small radii, which indicates the presence of a rapidly rotating gas in the innermost region of the galaxy. The inner-toouter velocity gradient ratio is $\Gamma>2$ and the intensity distribution along the line shows two symmetric peaks with respect to the center.

The $P V$ diagram of the Sa galaxy NGC 2179 (Fig. 5) can be considered the prototype of this class. As we showed in Bertola et al. (1998), the peculiar shape and intensity distribution of this $P V$ diagram can be modeled as a unique gaseous component that is rotating in the combined potential of a central mass concentration embedded in an extended stellar disk. Therefore the galaxies that exhibit this kind of $P V$ diagram (NGC 980, NGC 2179, and NGC 7782) are good candidates to host a CNKD rotating around a central mass concentration. They are ideal targets for HST spectroscopic follow-up to constrain the mass of the possible SMBH. A good estimate of this mass requires that the innermost kinematical points be within the radius of influence (e.g. Merritt \& Ferrarese 2001b). The three galaxies meet this criterion, since the expected angular extension of the radii of influence of their SMBHs are comparable to the pixel size of the Space Telescope Imaging Spectrograph $\left(\theta_{\bullet} \approx 00^{\prime \prime} 05\right)$. An increase in the velocity dispersion $\left(\sigma_{0} \gtrsim 150 \mathrm{~km} \mathrm{~s}^{-1}\right)$, associated with a large increase in the velocity gradient (as in NGC 980 


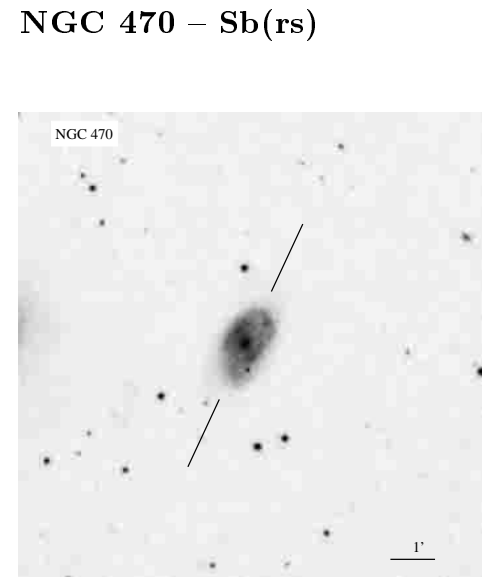

NGC $772-\mathrm{Sb}(\mathrm{s})$

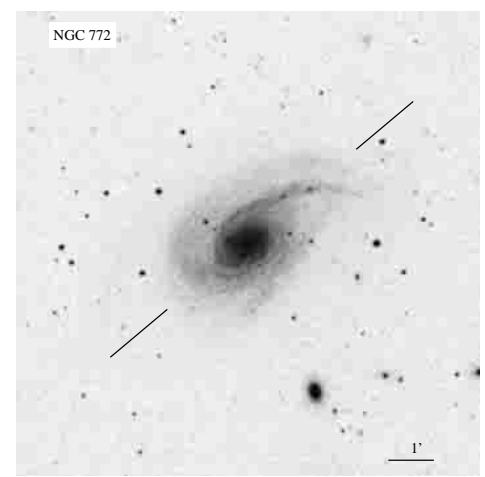

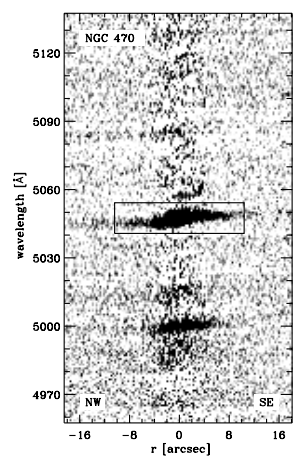
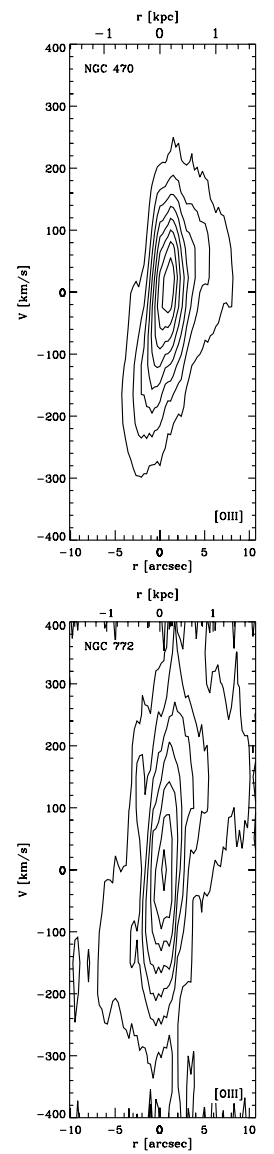

Fig. 2. Optical images, spectra and $P V$ diagrams of the sample galaxies. We show from left to right: a) an optical image of the galaxy taken from the Digitized Sky Survey with the slit position and angular scale superimposed. The orientation of the image is north up and east to the left. b) The galaxy spectrum after continuum removal with wavelength, radial distance from the nucleus, and orientation marked. Color cuts are chosen to show the fainter structures and the radial extension of the emission lines. In the INT and ESO spectra the nuclear continuum is the residual after subtraction of about $90 \%$ of the continuum. c) The galaxy $P V$ diagram derived from $[\mathrm{O}$ III $] \lambda 5007,[\mathrm{~N} \mathrm{II}] \lambda 6583$, and the $\mathrm{H} \alpha$ emission line for the spectra obtained at the MMT, INT, and 3.6-m ESO telescopes, respectively. In the $P V$ diagram the intensities of the plotted contours correspond to $5 \%, 15 \%, 25 \%, \ldots, 95 \%$ of the maximum emission-line intensity. The plotted region in the $P V$ diagram corresponds to the rectangular box marked in the galaxy spectrum. $P V$ diagrams are shown with the same scale for the observed radii and velocities, but we also indicate the distance from the center in kpc to aid comparison.

and NGC 2179) is expected in the presence of a nuclear mass concentration. It could result from differential motion within the aperture or from intrinsic turbulence in the gaseous disk. On the other hand, an increase in velocity dispersion that is not associated with an increase in the velocity gradient may indicate that the gas is not in a disk. However, a central mass concentration may still be the cause of this increase in the velocity dispersion.

Type II. This class of $P V$ diagram is characterized by a single velocity component which is in rigid-body rotation, as indicated by $\Gamma \approx 1 . \sigma_{0} / \sigma_{\text {out }} \approx 1$ and $F_{0} / F_{\text {out }} \approx 1$ are characteristic of these $P V$ diagrams too.

We consider the $P V$ diagram of the Sa galaxy NGC 5064 to be the prototypical example of this kind of $P V$ diagram (Fig. 5). In Bertola et al. (1998), we pointed out that in this galaxy either the unresolved Keplerian part of the gaseous disk does not result in a detectable contribution or the central mass concentration is lower than $5 \times 10^{7} M_{\odot}$. Therefore we suggest that galaxies exhibiting this type of
$P V$ diagram (see Table 4) may harbor low-mass SMBHs, although high spatial resolution spectroscopy and dynamical modelling of the stellar kinematics are required to distinguish this possibility from the effects of a peculiar gas distribution.

Type III. $P V$ diagrams of this type are characterized by an apparently broad nuclear emission-line component superimposed on a normal velocity curve. This results from the sharp increase of the line flux toward the center, as indicated by $F_{0} / F_{\text {out }}>1$.

The best example of this type of $P V$ diagram is that of the S0 NGC 2768 (Fig. 5). Most of the sample galaxies exhibit a $P V$ diagram belonging to this class because of a selection effect. They have been observed because of their strong emission lines.

The classification and the peculiarities of the $P V$ diagrams of all the sample galaxies are discussed in the appendix. 
NGC 949 - Sb(rs):
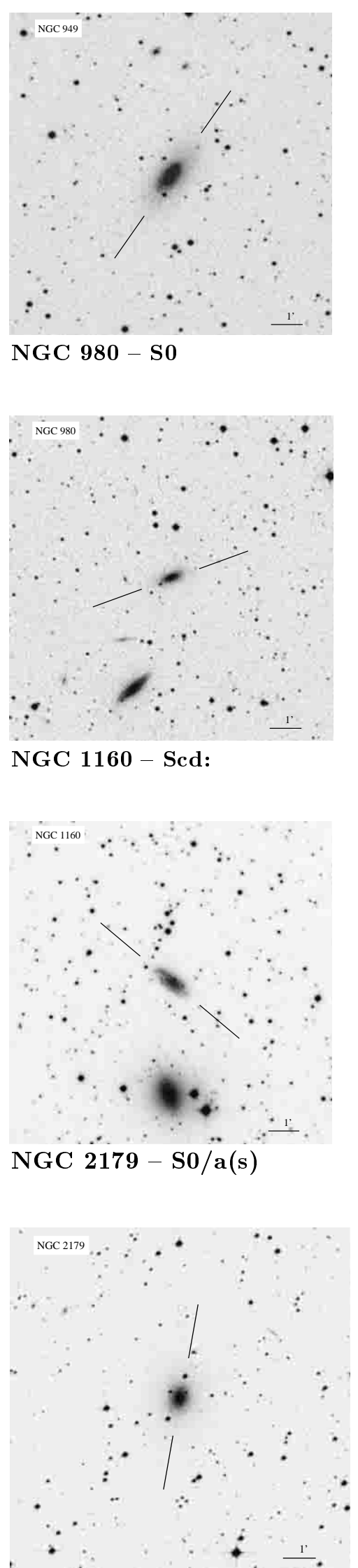
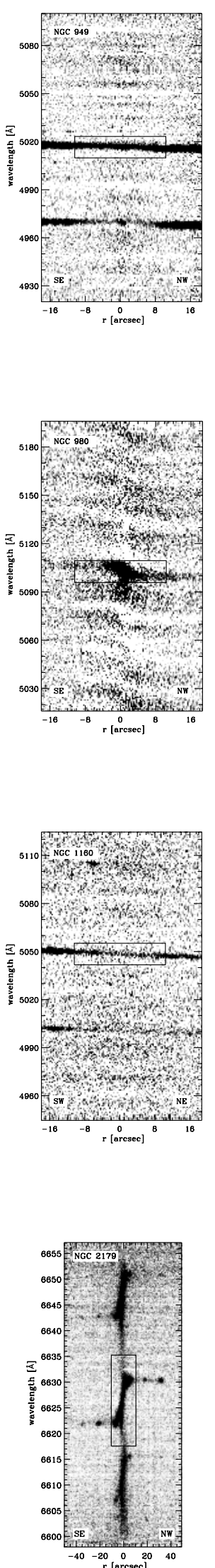
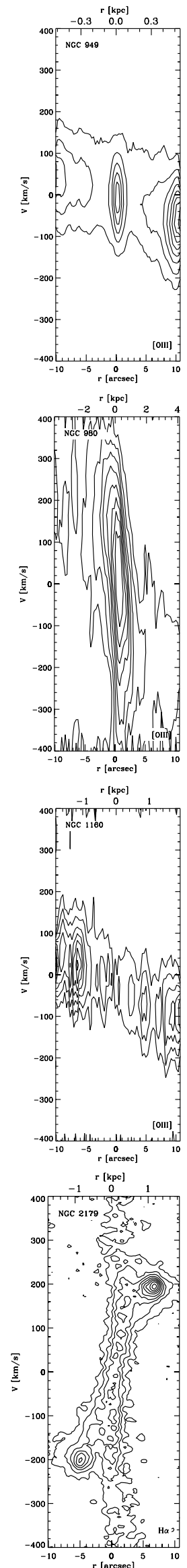

Fig. 2. continued. 

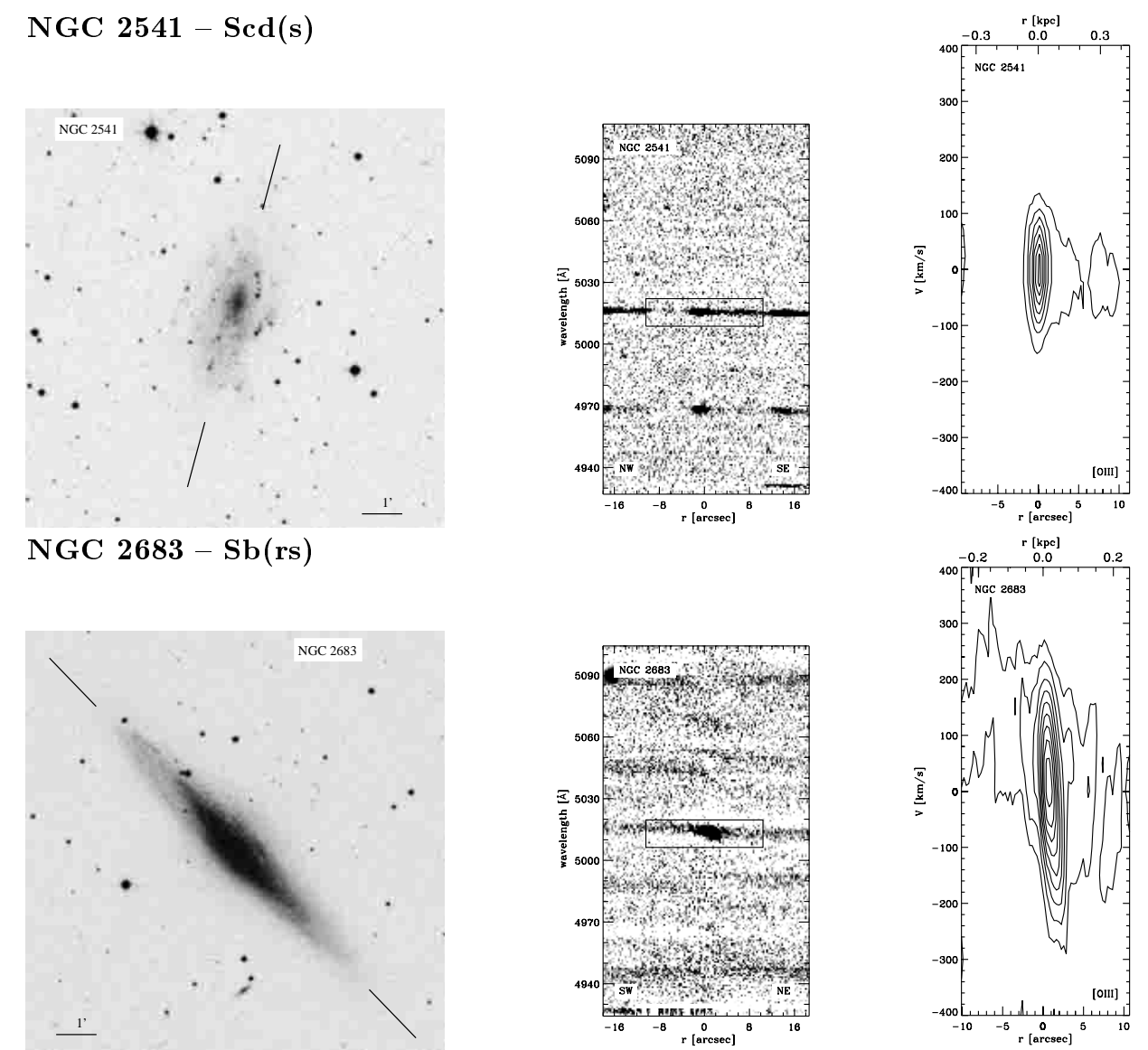

NGC $2768-\mathrm{S0}_{1 / 2}(6), \mathrm{E} 6:$
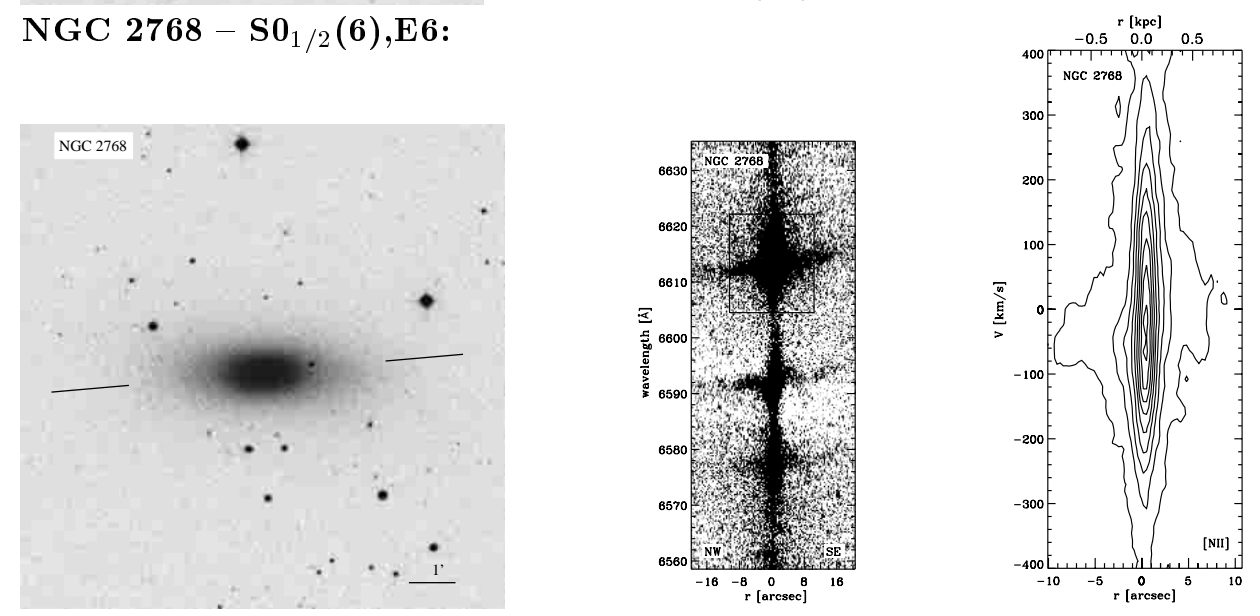

NGC 2815 - SBb(r):
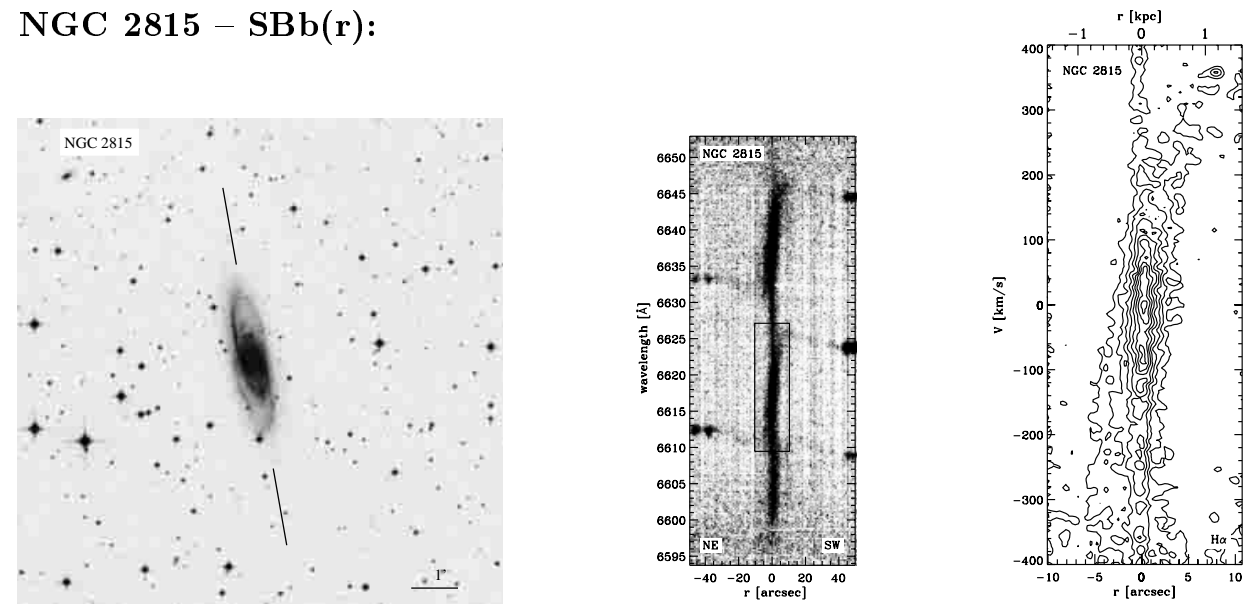

Fig. 2. continued. 

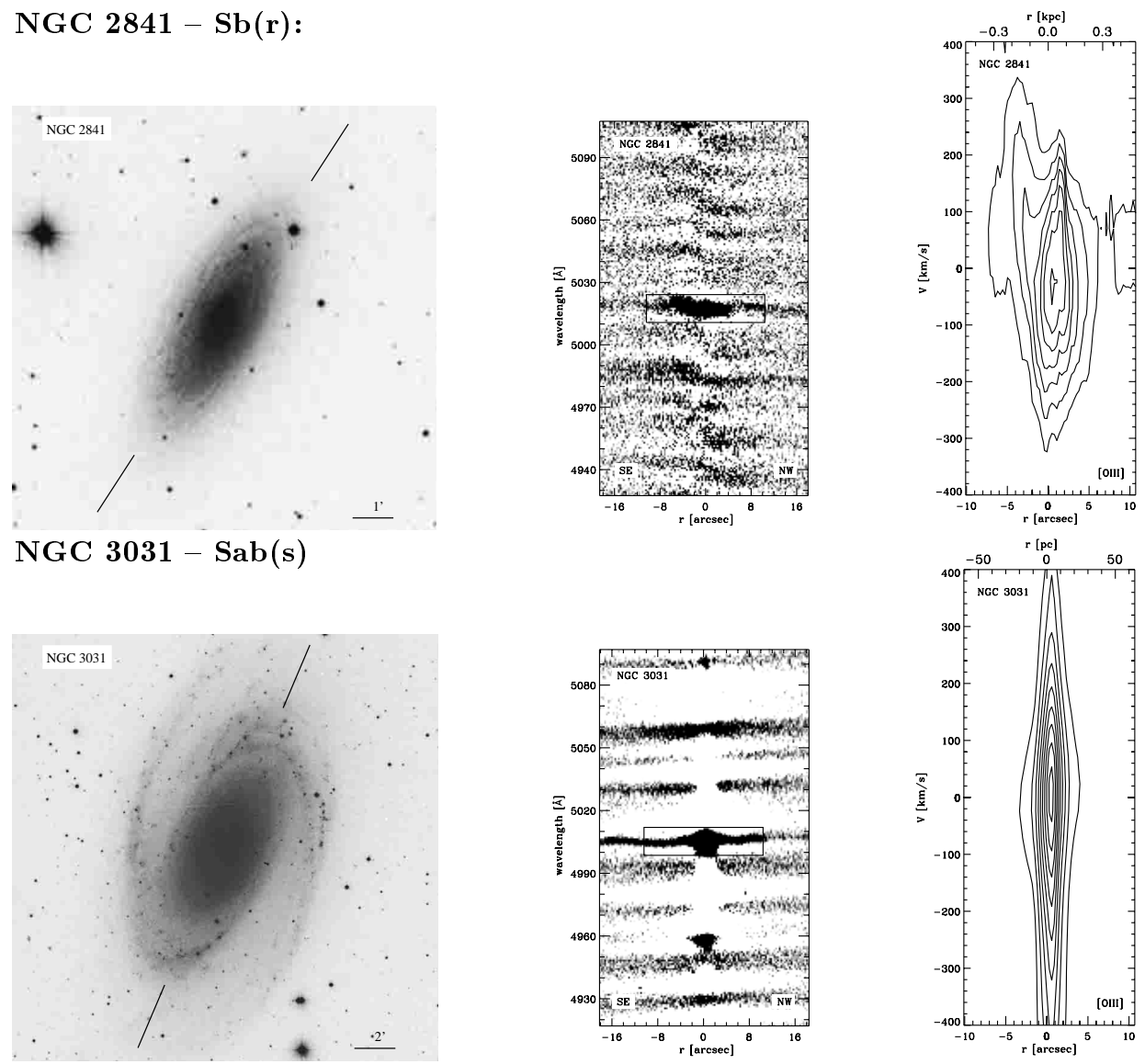

NGC 3281 - Sab(s)pec:
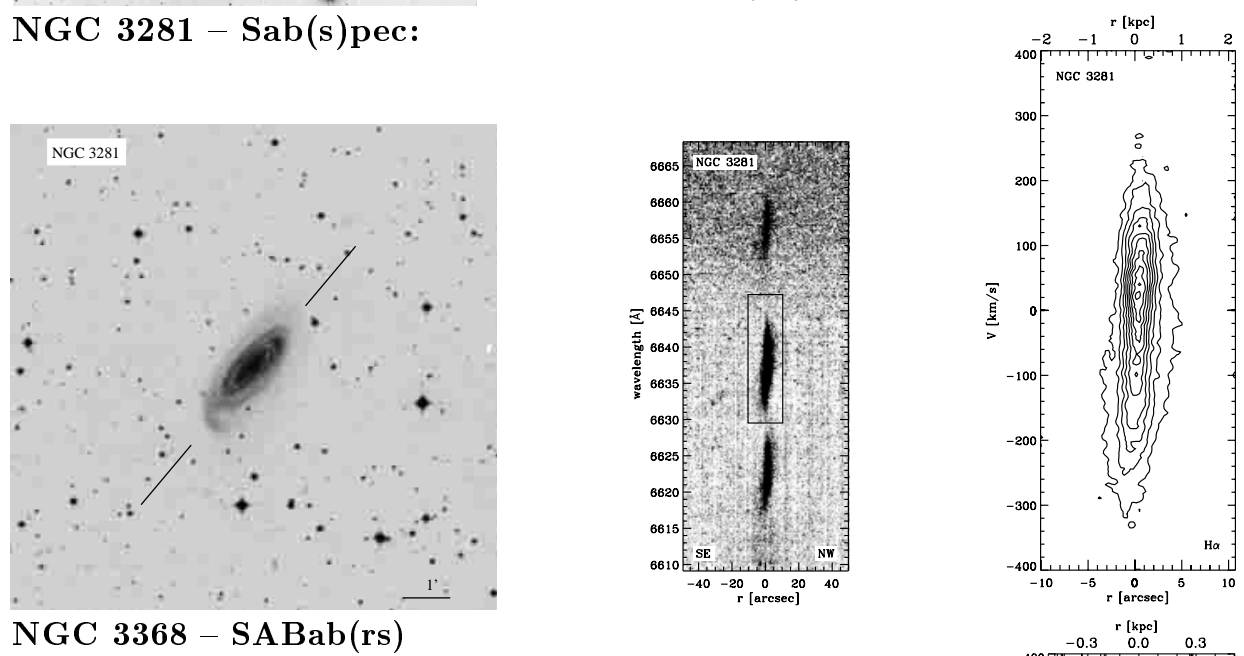

NGC 3368 - SABab(rs)
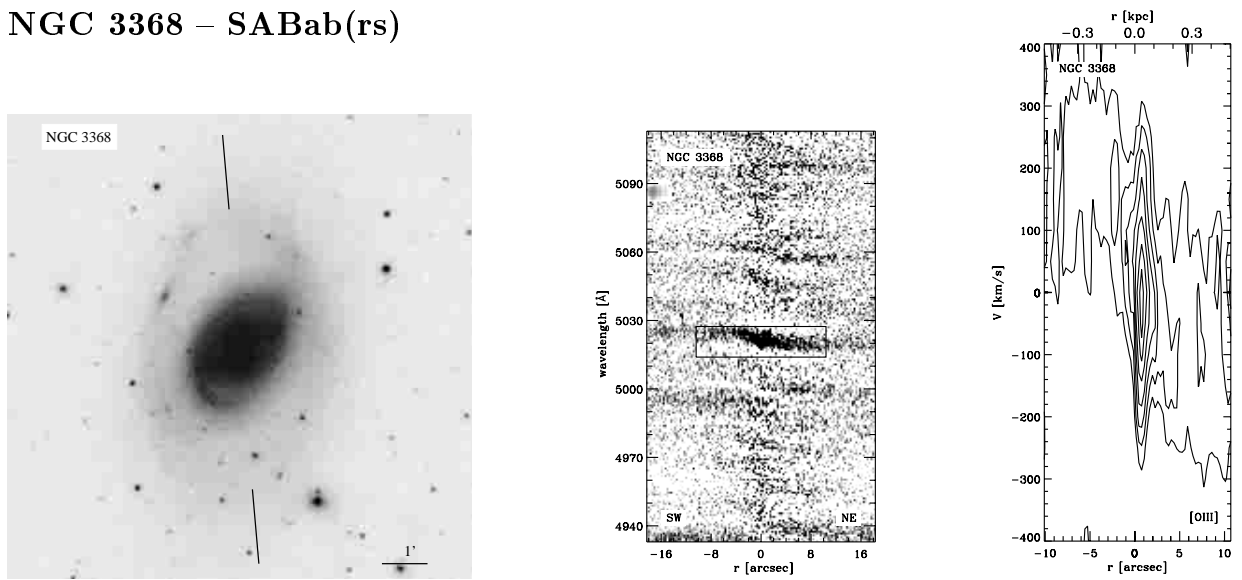

Fig. 2. continued. 

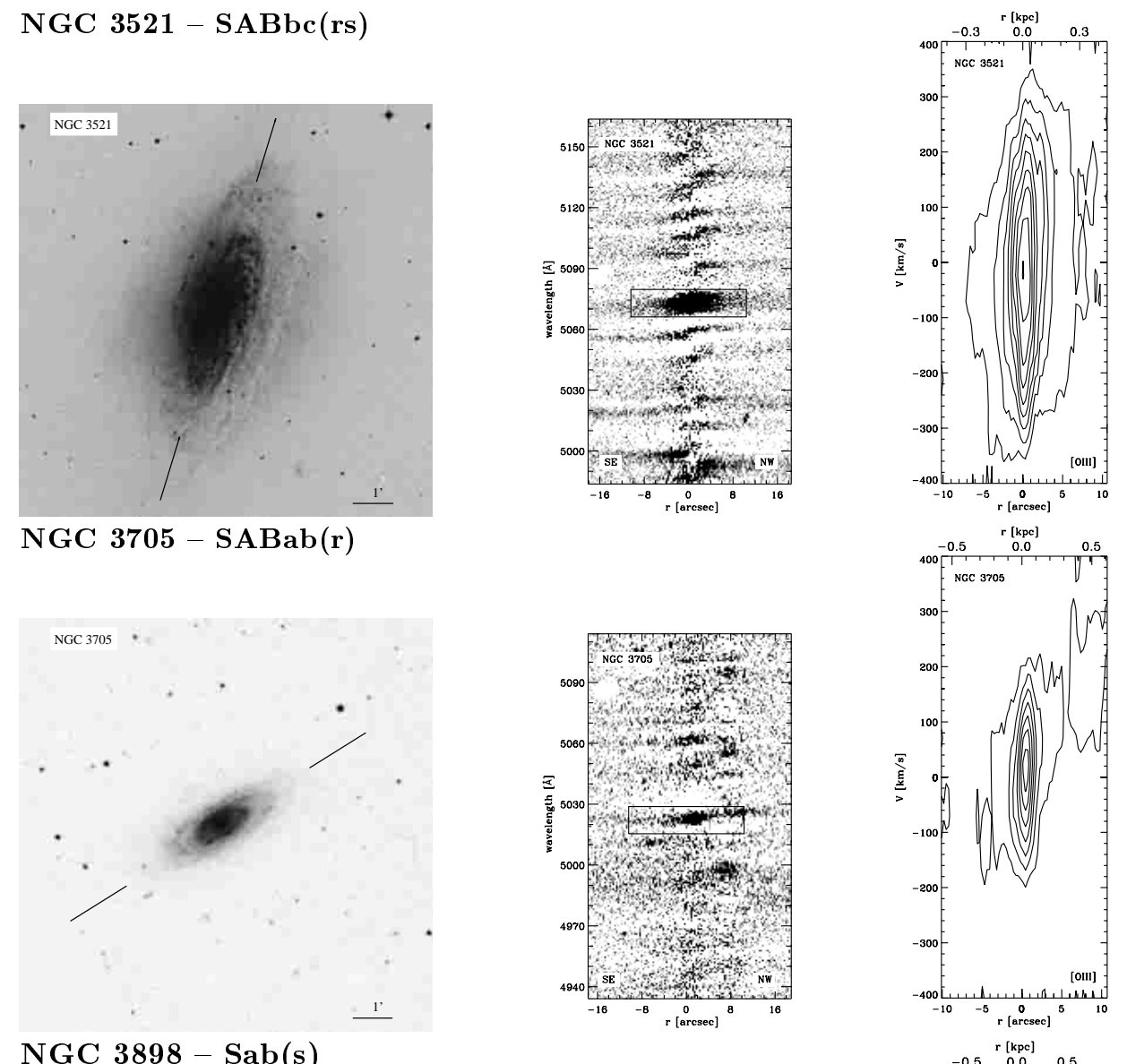

NGC 3898 - Sab(s)
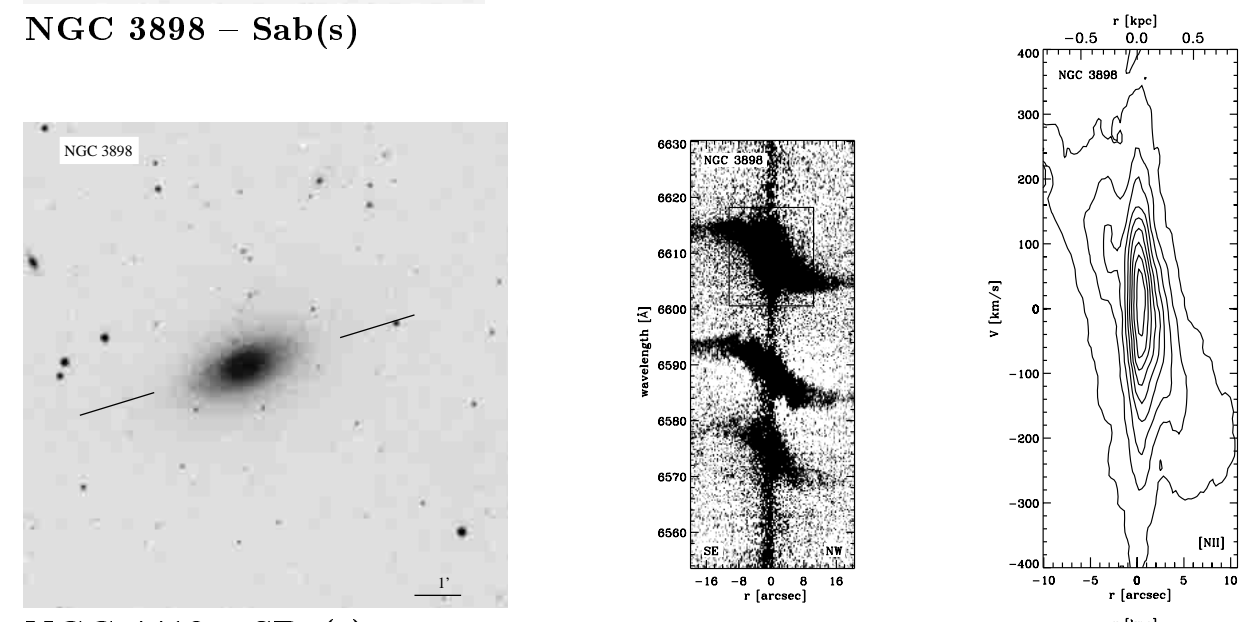

NGC 4419 - SBa(s)sp
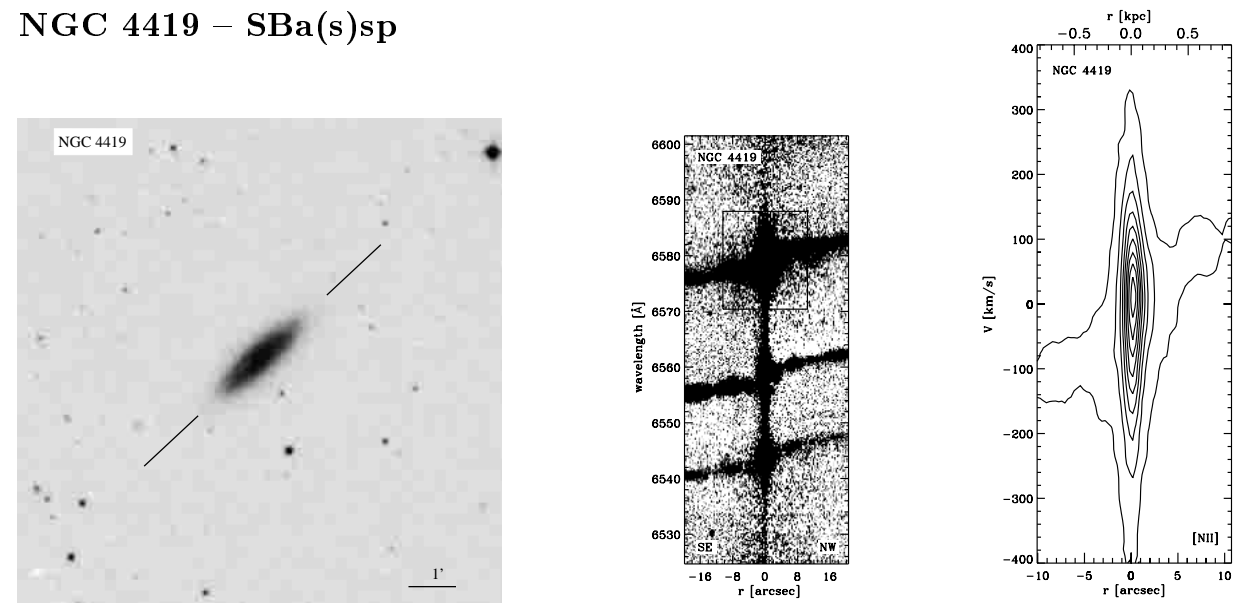

Fig. 2. continued. 
NGC 4698 - Sab(s)

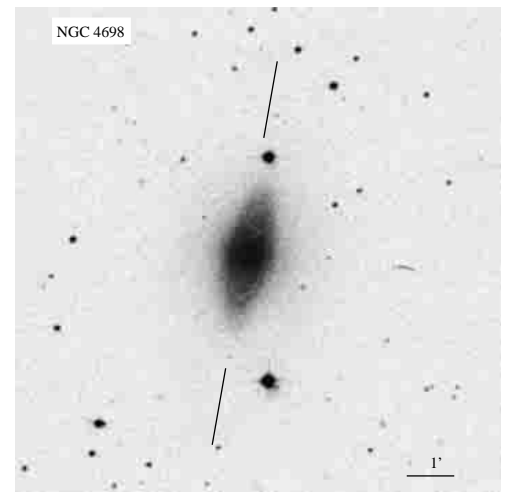

NGC 5064 - (R')Sab:
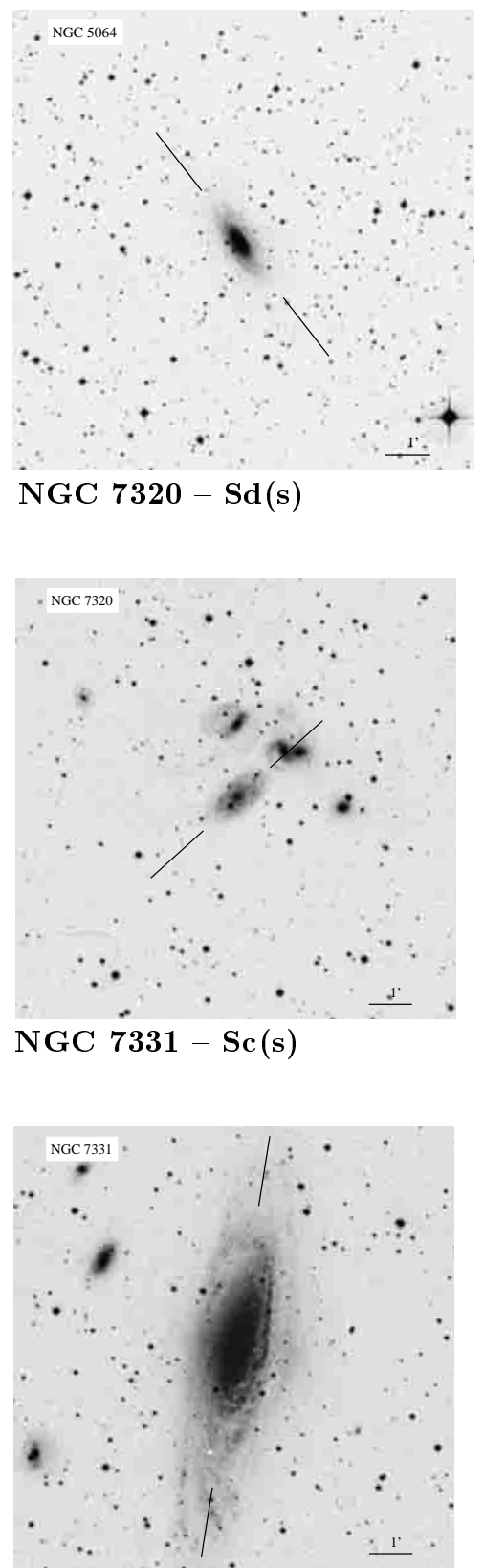
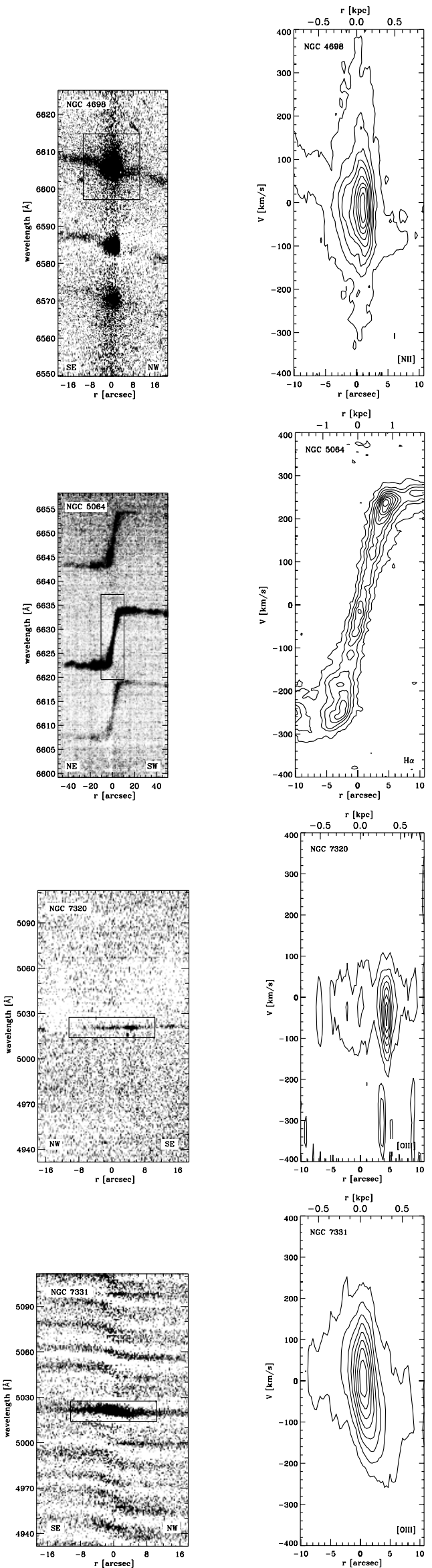

Fig. 2. continued. 
NGC $7782-$ Sc(s)

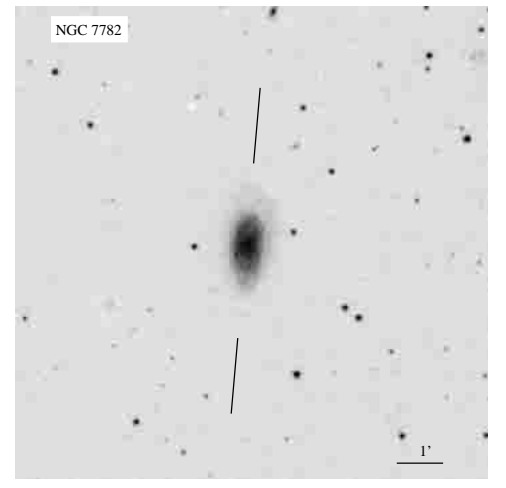

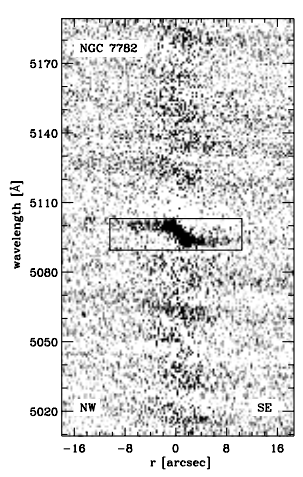

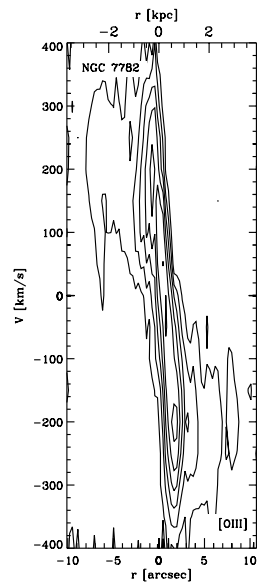

Fig. 2. continued.
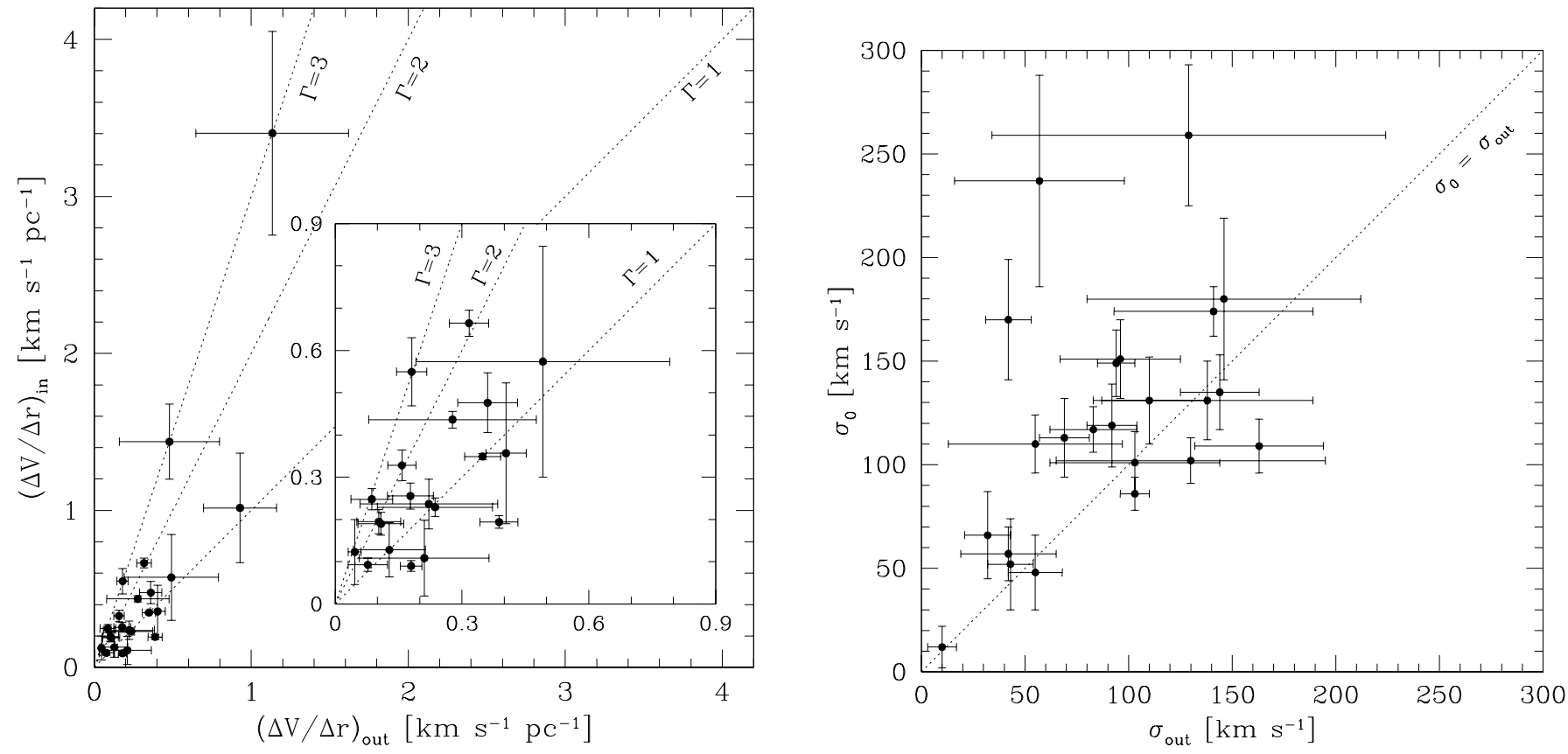

Fig. 3. Inner vs. outer velocity gradients (left panel) and central vs. outer velocity dispersions of the sample galaxies (right panel). Observed velocity gradients collected in Table 4 have been corrected for galaxy inclination and distance given in Table 1 .

\section{Discussion and conclusions}

In Bertola et al. (1998) we demonstrated that it is possible to detect the signature of a CNKD in the emission-line $P V$ diagrams obtained from ground-based spectroscopy of nearby galaxies. In addition, we showed that once properly modelled, this technique can lead to reliable upper-limits for the central mass concentration. Using these results, and to identify new galaxies hosting a CNKD, we obtained long-slit spectra along the major axes of 23 nearby disk galaxies, measuring the $P V$ diagrams of the ionized-gas components from the emission lines.

To achieve our goal, we analyzed these emission-line $P V$ diagrams by measuring the values of the velocity gradient, the velocity dispersion, and the integrated flux of the ionized gas at different radii, and by comparing the shape of the observed emission lines with that predicted for a thin disk of gas moving in circular orbits in the galaxy plane. This classification allowed us to recognize the possible presence of a CNKD in 3 of our objects. Recently, Rubin et al. (1997) and Sofue et al. (1998) discussed the kinematics of rapidly-rotating gas disks observed in the central few hundred parsecs of S0's and spiral galaxies. By combining our sample with their samples, we find that the $P V$ diagrams of 9 out 54 galaxies exhibit a $\Gamma>2$, which is indicative of a CNKD. The fact that in the majority of these cases CNKDs have not been observed means that not all of these galaxies enough gas detectable and rotating Keplerian orbits. Therefore, we estimated that the frequency of CNKDs, measured from ground-based spectroscopy of emission-line disk galaxies, is $\$ 20 \%$. This result is consistent with the findings of Sarzi et al. (2001), which are based on HST spectroscopy. Indeed, they found a gaseous disk with a well-ordered velocity field suitable 

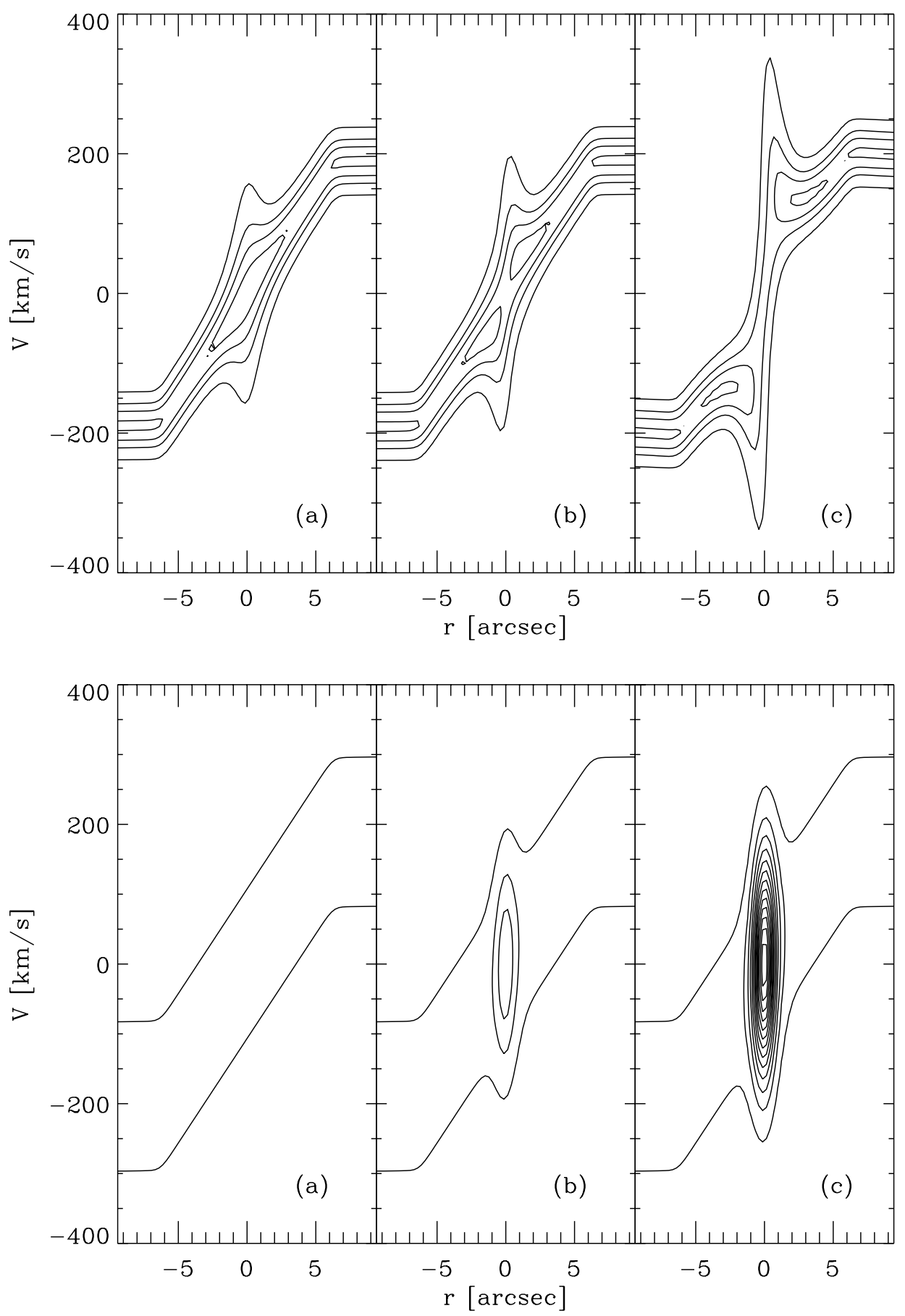

Fig. 4. Upper panels: the shapes of $P V$ diagrams as a function the central mass concentration. The three panels represent the emission lines of CNKDs rotating around central pointlike sources of a) $\left.M_{\bullet}=0 M_{\odot} ; \mathbf{b}\right) M_{\bullet}=10^{8} M_{\odot} ;$ and $\left.\mathbf{c}\right) M_{\bullet}=10^{9} M_{\odot}$. The $P V$ diagram in panel $\mathbf{c}$ ) is representative of Type I. Lower panels: the shape of $P V$ diagrams as a function of the intrinsic surface brightness of the gas. The three panels represent the emission lines of gaseous disks in rigid-body rotation with a projected velocity gradients $(\Delta V / \Delta r)_{\text {in }}=(\Delta V / \Delta r)_{\text {out }}=27 \mathrm{~km} \mathrm{~s}^{-1} \operatorname{arcsec}^{-1}$, observed velocity dispersions $\sigma_{0}=\sigma_{\text {out }}=100 \mathrm{~km} \mathrm{~s}$, and nuclear fluxes a) $F_{0}=F_{\text {out }}$; b) $F_{0}=20 \times F_{\text {out }}$; and c) $F_{0}=100 \times F_{\text {out }}$. The $P V$ diagrams in panels a) and c) are representative of Type II and III, respectively.

for dynamical modelling at HST resolution in only 4 of the 23 randomly selected emission-line disk galaxies they observed.

The analysis of ground-based $P V$ diagrams allows identification of those galaxies that are good candidates for hosting a CNKD rotating around a central mass concentration, and are therefore are good candidates for follow-up HST spectroscopy. It is worth noting that in this way it is possible to improve the present low success rate of HST programs aimed at estimating $\mathrm{SMBH}$ masses in nearby bulges by modelling nuclear gas kinematics. Although the SMBH mass hosted by the candidates 

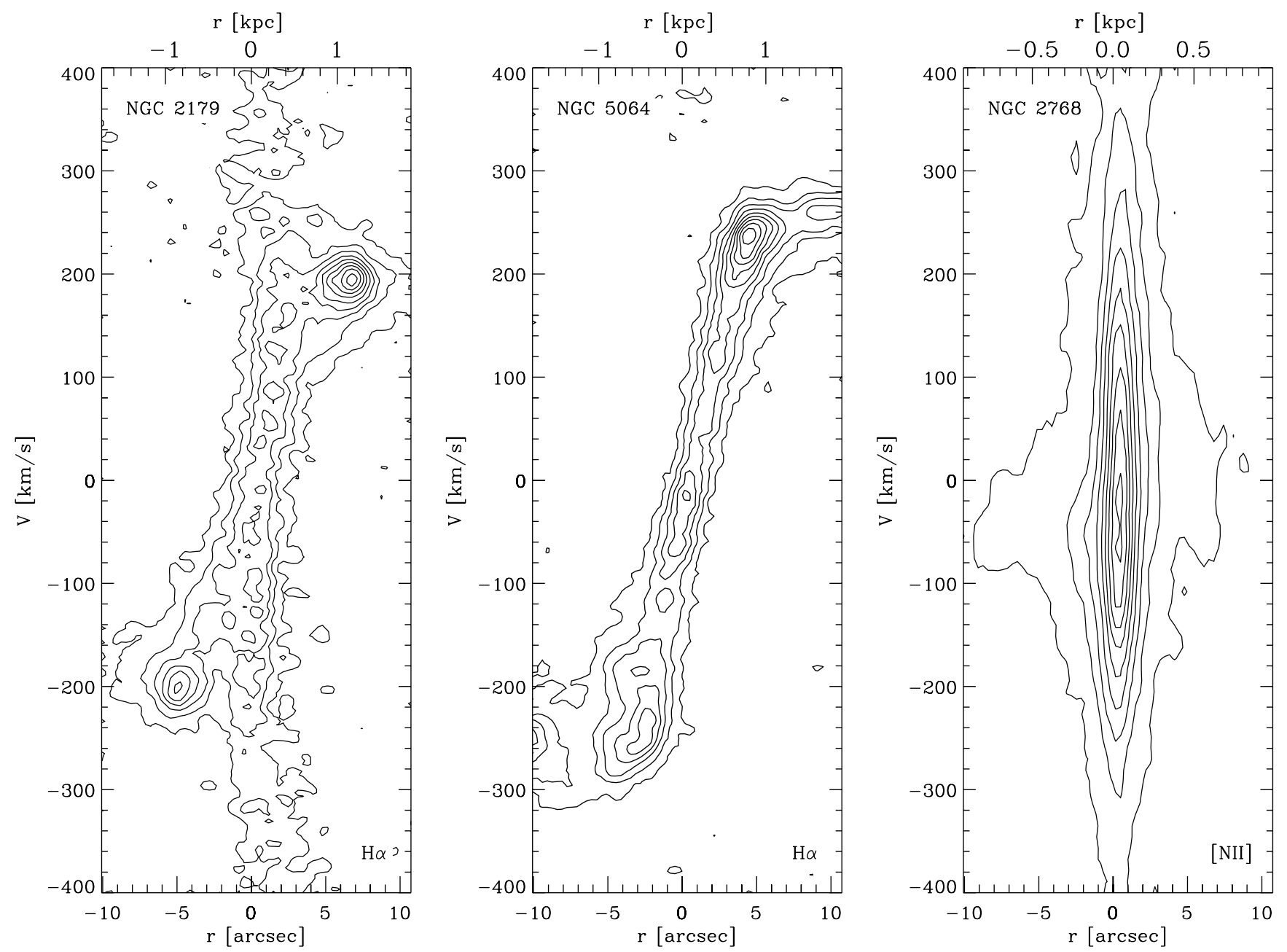

Fig. 5. Contour plots of the prototypical examples of the three types of $P V$ diagrams. Left panel: NGC 2179 (Type I). Middle panel: NGC 5064 (Type II). Right panel: NGC 2768 (Type III).

selected by ground-based observations are expected to lie in the high-mass end of the $M_{\bullet}-\sigma$ diagram because of the resolution limits imposed on their $P V$ diagrams by seeing, these candidates are required to elucidate the relationship for disk galaxies, which are underrepresented relative to elliptical galaxies in the sample of galaxies studied so far.

Acknowledgements. We thank Betsy Gillespie for reading the manuscript. This research has made use of the Lyon-Meudon Extragalactic Database (LEDA) and Digitized Sky Survey (DSS).

\section{Appendix A: Notes on individual galaxies}

The $P V$ diagrams of the sample galaxies are plotted in Fig. 2 and their classifications according to the criteria proposed in Sect. 3.3 are given in Table 4. In this section, we describe the most important properties of the sample galaxies and discuss the classification of their $P V$ diagrams. The values of the velocities and the velocity dispersions include no corrections for inclination.

NGC 470. This intermediate-type spiral hosts either two nested bars (Wozniak et al. 1995) or a bar with a triaxial bulge (Friedli et al. 1996). The velocity gradient and velocity dispersion of the [O III] $\lambda 5007$ line do not significantly change moving from the nuclear to the outer regions, while its integrated flux increases toward the center. We classify the $P V$ diagram of NGC 470 as Type III.

NGC 772. According to the dynamical modelling of Pignatelli et al. (2001), the velocity of the ionized gas traces the circular speed in gravitational equilibrium. The $P V$ diagram of NGC 772 derived from the [O III] $\lambda 5007$ emission line is classified as Type III since the integrated flux of the emission line in the nucleus is greater than that measured in the outer regions.

NGC 949. As in the previous case, also the Type III classification of the $P V$ diagram of NGC 949 results from the increase of the integrated flux of the $[\mathrm{O}$ III $] \lambda 5007$ line toward the center.

NGC 980. In the $P V$ diagram of this S0 galaxy, a tilted and bright component appears to be superimposed on a normal rotation curve. The increase of both the velocity gradient and the velocity dispersion toward the center are indicative of the kinematics of a CNKD and give this $P V$ 
diagram its Type I classification. This is also the case for NGC 2179 and NGC 7782.

NGC 1160. The $P V$ diagram of this Scd spiral is characterized by a constant velocity gradient and a constant integrated flux for the $[\mathrm{O}$ III $] \lambda 5007$ line. This makes its Type II classification straightforward.

NGC 2179. The $P V$ diagram of NGC 2179 is the prototype of the Type I class. The two-dimensional shapes of the emission lines are such that they gives the erroneous impression of two distinct velocity components. One apparent velocity component has the form of a highly tilted straight line rising from zero velocity in the galaxy center (i.e., a faster-rising rotation curve). The other component is a less-tilted straight line (i.e., a slower-rising rotation curve) superimposed on the first. Both lines naively appear to imply solid-body rotation in the inner parts of this galaxy; both lines culminate as the radius increases to almost the same maximum velocity; the slower-rising rotation curve shows a flat portion in its outer regions. Rather than being of two different physical origins, we have shown that properly accounting for the seeing, slit width, and pixel size effects, these two apparently solidbody rotation curves can be modelled as the velocity field of a thin gaseous disk rotating in the combined gravitational potential of central point-like mass and an extended stellar disk (see Bertola et al. 1998 for details).

NGC 2541. The bright central component in this $P V$ diagram is characterized by the same velocity dispersion measured in the outer parts of the disk. This is typical of Type III diagrams.

NGC 2683. According to Merrifield \& Kuijken (1999), the PV diagram of NGC 2683 has a "figure-of-eight" shape produced by the presence of two kinematically distinct gaseous components. This feature is barely visible in our $P V$ diagram because of the lower $S / N$ ratio of the spectrum. Although the properties of the $P V$ diagram of NGC 2683 are similar to those of NGC 980, NGC 2179 and NGC 7782, it does not warrant a Type I classification. Indeed in NGC 2683 we are observing two gas components which are spatially distinct and superimposed along the line of sight because of the high inclination of the galaxy $\left(i=78^{\circ}\right)$. They are generated by the presence of a non-axisymmetric potential (Kuijken \& Merrifield 1995; Bureau \& Athanassoula 1999).

This is not the case for NGC 980, NGC 2179 and NGC 7782 , which are less inclined $\left(i=58^{\circ}, 47^{\circ}\right.$ and $58^{\circ}$, respectively) and unbarred, exhibiting unique gaseous components.

NGC 2768. The presence of a definite outer envelope with subtle dust patches surrounding the bulge (see panels 38 and 53 in CAG) supports the S0 classification of this galaxy, which appears as an E6* in RC3. The kinematical decoupling between the ionized gas and the stars, detected by Bertola et al. (1992), has been interpreted as a result of the presence of gas orbiting in a polar ring
(Möllenhoff et al. 1992; Fried \& Illinghworth 1994). The inner velocity gradient is higher than the outer one which is one of the lowest we measured $\left((\Delta V / \Delta r)_{\text {out }}=\right.$ $\left.0.09 \mathrm{~km} \mathrm{~s}^{-1} \mathrm{pc}^{-1}\right)$. The constant velocity dispersion and the steep increase of [N II] $\lambda 6583$ flux in the center imply a Type III classification for this $P V$ diagram.

NGC 2815. The presence of the spectrum of broad emission lines in the nuclear region of NGC 2815 with wings which are very close each to other makes the subtraction of the galaxy continuum critical. Indeed, residual continuum is still visible in the $P V$ diagram derived from the $\mathrm{H} \alpha$ line; it gives the erroneous impression that the $P V$ diagram shape is similar to that of NGC 2179 . We classify this $P V$ diagram as Type III class because of its constant velocity gradient and large central-to-outer integrated-flux ratio.

NGC 2841. According to Sil'Chenko et al. (1997) the ionized gas is rotating orthogonally with respect to the galaxy plane in the inner $5^{\prime \prime}$. Alternatively, Sofue et al. (1998) reported that the central portion of the $P V$ diagram derived from the $\mathrm{H} \alpha$ and [N II] $\lambda 6583$ lines is slightly tilted in the direction of the galactic rotation, suggesting the presence of a rapidly rotating nuclear disk. Our $P V$ diagram exhibits a complex and asymmetric shape that could be related to these different kinematic components. However, we do not measure a significant variation of the velocity gradient or the velocity dispersion with radius. The line flux increases slightly toward the center. These features are similar to those of $P V$ diagrams included in the Type III class, and make it difficult to associate with the central component to a fast-rotating disk as by indicated Sofue et al. (1998).

NGC 3031. HST H $\alpha$ imaging reveals the presence of a nuclear gaseous disk (Dereveux et al. 1997) similar in size and shape to the CNKD of M 87 (see Macchetto et al. 1997 and references therein). The disk is rotating around a SMBH with $M_{\bullet}=3 \times 10^{6} M_{\odot}$, according to determinations based on stellar kinematics (Bower et al. 1996) and broad-line emission (Ho et al. 1996). The spatial and spectral resolution of our spectrum allow us only to detect the presence of a broad and bright central component in the $P V$ diagram. In fact, it exhibits the highest central-toouter integrated-flux ratio of our sample, which warrants a Type III classification. From the available spectrum, it is difficult to claim that NGC 3031 is hosting a CNKD even though we measure a remarkably large $(\Delta V / \Delta r)_{\text {in }}$ $\left(=3.4 \mathrm{~km} \mathrm{~s}^{-1} \mathrm{pc}^{-1}\right)$ and a large inner-to-outer velocitygradient ratio $(\Gamma=3.0)$.

NGC 3281. The ionized-gas kinematics measured by Rubin et al. (1985) and Corsini et al. (1999) extends out to about $50^{\prime \prime}$ from the nucleus but in our spectrum the emission is confined in the innermost $5^{\prime \prime}$. The inner velocity gradient is steeper than the outer one and this early-type spiral has one of the highest central-to-outer flux ratios of the whole sample. Even if the emission is not extended we 
consider the $P V$ diagram of NGC 3281 to be of Type III because of its intense nuclear emission.

NGC 3368. From NIR photometry, Jungwiert et al. (1997) identified a possible double-barred structure within this Sab spiral. Although the $P V$ diagram seems to have a two-component structure, the constant velocity gradient and steep increase of the integrated flux of the [O III] $\lambda 5007$ line toward the center suggest a Type III classification.

NGC 3521. The $P V$ diagram of this intermediatetype spiral has been recently measured by Sofue et al. (1998) from the $\mathrm{H} \alpha$ and the $[\mathrm{N} \mathrm{II}] \lambda 6583$ emission lines. They interpreted the central component observed in the [N II] $\lambda 6583$ line as an indication of the presence of a fast rotating gaseous disk in the nucleus. We suggest that this feature, which is clearly visible also in the PV diagram we derived from the [O III] $\lambda 5007$ emission line, results from the increase of the line flux rather than the velocity gradient. Morever, the velocity dispersion does not change with radius. Therefore it is Type III diagram.

NGC 3705. The Type III classification of this $P V$ diagram mostly results from the centrally-peaked radial profile of the integrated flux of the [O III] $\lambda 5007$ line, which gives the impression of a steep central component superimposed on a slowly-rotating component.

NGC 3898. The ionized-gas distribution and kinematics of this Sa galaxy have recently been studied in detail by Pignatelli et al. (2001). They found that in the innermost region $\left(|r| \lesssim 8^{\prime \prime}\right)$ of NGC 3898, the ionized gas is rotating more slowly than the circular velocity predicted by dynamical modelling based on stellar kinematics and photometry. The fingerprint of such a "slowly-rising" rotation curve (according to Kent 1988 definition) can be recognized in the decrease of the velocity gradient at smaller radii. The two-component shape of the PV diagram results from the bright nuclear emission and not the increase of velocity gradient or the velocity dispersion. This is a Type III PV diagram and its similarity to the $P V$ diagram of NGC 4419 is remarkable.

NGC 4419. The spectrum and consequently the $P V$ diagram of NGC 4419 are similar to those of NGC 3898. The spectra show the same strong and broad $\mathrm{H} \alpha$ absorption and the $P V$ diagrams are characterized by the same bright central component. They have also similar inner-to-outer velocity gradient and dispersion ratios and both belong to the Type III class. As NGC 3898 also NGC 4419 is one of the bulge-dominated spirals displaying a slowly-rising rotation curve of the ionized gas discussed by Kent (1988).

NGC 4698. This Sa galaxy shows a remarkable orthogonal geometrical and kinematical decoupling between the inner portion of the bulge and galaxy disk (Bertola et al. 1999). The asymmetric shapes of the $\mathrm{H} \alpha$ and $[\mathrm{N} \mathrm{II}]$ lines are seen at a simple visual inspection of the spectrum, and they are more evident in the PV diagram obtained from the $[\mathrm{N}$ II $] \lambda 6583$ line. Although we measured an increase of the velocity gradient toward the center, we note that NGC 4698 has the shallowest outer gradient of all the sample galaxies $\left((\Delta V / \Delta r)_{\text {out }}=0.05 \mathrm{~km} \mathrm{~s}^{-1} \mathrm{pc}^{-1}\right)$. This gradient corresponds to the central plateau measured in the ionized-gas rotation curve by Bertola \& Corsini (2000). The Type III classification has been assigned to this $P V$ diagram on the basis of its high central-to-outer integrated-flux ratio.

NGC 5064. The $P V$ diagram of NGC 5064 is the prototype of the Type II class. It is useful to compare the emission-line spectrum of NGC 5064 to that of NGC 2179, because both spectra have been obtained with same setup and observing conditions. In contrast with NGC 2179, the emission-line spectrum of NGC 5064 does not show any peculiar features; there is only one component in the central region. The velocity increases linearly with radius until it reaches about $200 \mathrm{~km} \mathrm{~s}^{-1}, 4^{\prime \prime}$ from the center. The velocity dispersion and the integrated flux of the $\mathrm{H} \alpha$ line remain almost constant in this radial range.

NGC 7320. The spectrum we obtained for this late-type spiral, which belongs to Stephan's Quintet is of poor quality. The [O III] $\lambda 5007$ line shows a bright knot at about $5^{\prime \prime}$ from the center resulting in the observed $F_{0} / F_{\text {out }}=0.3$. The Type II classification of the PV diagram is based on the constant inner-to-outer velocity gradient and velocity dispersion.

NGC 7331. The presence of a $\operatorname{SMBH}\left(M_{\bullet} \sim 10^{8} M_{\odot}\right)$ in the center of NGC 7331 has been debated by different authors (Afanasiev et al. 1989; Bower et al. 1993; Mediavilla et al. 1997; Sil'Chenko 1999). The debate centers on observations of the distribution and kinematics of ionized gas. Our $P V$ diagram is similar to that of NGC 772 . We measure an increase of the integrated flux of the $[\mathrm{O}$ III $] \lambda 5007$ line at smaller radii, along with constant velocity gradient and to slight increase of the velocity dispersion. The $P V$ diagram of NGC 7331 is of Type III.

NGC 7782. The inner region of this $P V$ diagram is characterized by a sharp increase of the velocity gradient, as confirmed by the large inner-to-outer velocity-gradient ratio we measure. The $[\mathrm{O}$ III $] \lambda 5007$ line exhibits a bright nuclear component and it velocity dispersion rapidly decreases with radius. The properties of the $P V$ diagram of NGC 7782 are close to those of NGC 2179 leading to the Type I classification. The nuclear ionized-gas kinematics of NGC 7782 is indicative of a CNKD.

\section{References}

Afanasiev, V. L., Morozov, A., \& Levi, V. 1989, in Extranuclear Activity in Galaxies, ed. E. J. A. Meurs, \& R. A. E. Fosbury (Garching bei München: ESO), 369

Bender, R., Saglia, R. P., \& Gerhard, O. E. 1994, MNRAS, 269, 785

Bertola, F., \& Corsini, E. M. 2000, in Dynamics of Galaxies: from the Early Universe to the Present, ed. F. Combes, G. A. Mamon, \& V. Charmandaris (San Francisco: ASP), ASP Conf. Ser., 197, 115 
Bertola, F., Buson, L. M., \& Zeilinger, W. W. 1992, ApJ, 401, L79

Bertola, F., Cinzano, P., Corsini, E. M., Rix, H.-W., \& Zeilinger, W. W. 1995, ApJ, 448, L13

Bertola, F., Cappellari, M., Funes, J. G., et al. 1998, ApJ, 509, L93

Bertola, F., Corsini, E. M., Vega Beltrán, J. C., et al. 1999, ApJ, 519, L127

Bower, G. A., Richstone, D. O., Bothun, G. D., \& Heckman, T. M. 1993, ApJ, 402, 76

Bower, G. A., Wilson, A. S., Heckman, T. M., \& Richstone, D. O. 1996, AJ, 111, 1901

Bureau, M., \& Athanassoula, E. 1999, ApJ, 522, 686

Corsini, E. M., Pizzella, A., Sarzi, M., et al. 1999, A\&A, 342, 671

de Vaucouleurs, G., de Vaucouleurs, A., Corwin, H. G. Jr., et al. 1991, Third Reference Catalogue of Bright Galaxies (New York: Springer-Verlag) (RC3)

Devereux, N., Ford, H., \& Jacoby, G. 1997, ApJ, 481, L71

de Zeeuw, P. T. 2001, in Black Holes in Binaries and Galactic Nuclei, ed. L. Kaper, E. P. J. van den Heuvel, \& P. A. Woudt (Berlin: Springer-Verlag), 78

Ferrarese, L., \& Merritt, D. 2000, ApJ, 539, L9

Ferrarese, L., Pogge, R. W., Peterson, B. M., et al. 2001, ApJ, 555, L79

Freedman, W. L., Madore, B. F., Mould, J. R., et al. 1994, Nature, 371, 757

Fried, J. W., \& Illingworth, G. D. 1994, AJ, 107, 992

Friedli, D., Wozniak, H., Rieke, M., Martinet, L., \& Bratschi, P. 1996, ApJS, 118, 461

Gebhardt, K., Bender, R. B., Dressler, A., et al. 2000a, ApJ, 539, L13

Gebhardt, K., Kormendy, J., Ho, L. C., et al. 2000b, ApJ, 543, L5

Guthrie, B. N. G. 1992, A\&AS, 93, 255

Ho, L. C., 1999, in Observational Evidence for Black Hole in the Universe, ed. S. K. Chakrabati (Dodrecht: Kluwer Academic Publishers), 157

Ho, L. C., Filippenko, A. V., \& Sargent, W. L. W. 1996, ApJ, 462, 183

Ho, L. C., Filippenko, A. V., \& Sargent, W. L. W. 1997, ApJS, 112,315

Jungwiert, B., Combes, F., \& Axon, D. J. 1997, A\&AS, 125, 479

Kent, S. M. 1988, AJ, 96, 514

Kormendy, J. 2001, in Galaxy Disks and Disk Galaxies, ed. J. G. Funes, \& E. M. Corsini (San Francisco: ASP), ASP Conf. Ser., 230, 247
Kormendy, J., \& Gebhardt, K. 2001, in The 20th Texas Symposium on Relativistic Astrophysics, ed. H. Martel, \& J. C. Wheeler (New York: AIP), 586, 363

Kormendy, J., \& Richstone, D. 1995, ARA\&A, 33, 581

Kuijken, K., \& Merrifield, M. R. 1995, ApJ, 443, L13

Macchetto, F., Marconi, A., Axon, D. J., et al. 1997, ApJ, 489, 579

Magorrian, J., Tremaine, S., Richstone, D. et al. 1998, AJ, 115, 2285

Marzke, R. O., da Costa, L. N., Pellegrini, P. S., Willmer, C. N. A., \& Geller, M. J. 1998, ApJ, 503, 617

McLure, R. J., \& Dunlop, J. S. 2001, MNRAS, 327, 199

McLure, R. J., \& Dunlop, J. S. 2002, MNRAS, in press [astro-ph/0108417]

Mediavilla, E., Arribas, S., Garcia-Lorenzo, B., \& del Burgo, C., 1997, ApJ, 488, 682

Merrifield, M. R., \& Kuijken, K. 1999, A\&A, 345, L47

Merritt, D., \& Ferrarese, L. 2001a, ApJ, 547, 140

Merritt, D., \& Ferrarese, L. 2001b, in The Central Kpc of Starbursts and AGN, ed. J. H. Knapen, J. E. Beckman, I. Shlosman, \& T. J. Mahoney (San Francisco: ASP), ASP Conf. Ser., 249, 335

Möllenhoff, C., Hummel, E., \& Bender, R. 1992, A\&A, 255, 35

Pignatelli, E., Corsini, E. M., Vega Beltrán, J. C., et al. 2001, MNRAS, 323, 188

Rubin, V. C., Burstein, D., Ford, W. K. Jr., \& Thonnard, N. 1985, ApJ, 289, 81

Rubin, V. C., Kenney, J. D. P., \& Young, J. S. 1997, AJ, 113, 1250

Sandage, A., \& Bedke, J. 1994, The Carnegie Atlas of Galaxies (Washington: Carnegie Institution of Washington and the Flintridge Foundation) (CAG)

Sandage, A., \& Tammann, G. A. 1981, A Revised ShapleyAmes Catalog of Bright Galaxies (Washington: Carnegie Institution of Washington) (RSA)

Sarzi, M., Rix, H.-W., Shields, J. C., et al. 2001, ApJ, 550, 65

Sil'Chenko, O. K. 1999, AJ, 118, 186

Sil'Chenko, O. K., Vlasyuk, V. V., \& Burenkov, A. N. 1997, A\&A, 326, 941

Sofue, Y., Tomita, A., Tutui, Y., Honma, M., \& Takeda, Y. 1998, PASJ, 50, 427

Vega Beltrán, J. G., Pizzella, A., Corsini, E. M., et al. 2001, A\&A, 374, 394

Véron-Cetty, M.-P., \& Véron, P. 1986, A\&AS, 66, 335

Wozniak, H., Friedli, D., Martinet, L., Martin, P., \& Bratschi, P. 1995, ApJS, 111, 115

Zeilinger, W. W., Vega Beltrán, J. C., Rozas, M., et al. 2001, Ap\&SS, 276, 643 